\title{
Analysis and Realization of Sampling Strategy in Coordinate Metrology
}

\author{
Syed Hammad Mian (D), Abdulrahman Al-Ahmari, and Hisham Alkhalefah \\ Raytheon Chair for Systems Engineering (RCSE Chair), Advanced Manufacturing Institute, King Saud University, Riyadh, Saudi Arabia \\ Correspondence should be addressed to Syed Hammad Mian; smien@ksu.edu.sa
}

Received 11 February 2019; Accepted 17 June 2019; Published 18 July 2019

Academic Editor: Anna M. Gil-Lafuente

Copyright (C) 2019 Syed Hammad Mian et al. This is an open access article distributed under the Creative Commons Attribution License, which permits unrestricted use, distribution, and reproduction in any medium, provided the original work is properly cited.

\begin{abstract}
The stringent customer demands and competitive market emphasize the importance of efficient and effective inspection in industrial metrology. Therefore, the implementation of an appropriate sampling strategy, i.e., the number of points and their distribution, has become very important in the inspection process using a coordinate measuring machine. Moreover, the quality of inspection results has frequently been influenced by sampling plan as well as workpiece size and surface characteristics. It has been an indispensable problem in the present-day measurement processes. Thus, this paper investigates various sample sizes and different point distribution algorithms that can be employed in the evaluation of form error. The effect of specimen size and surface quality on the sampling strategy has also been investigated. Furthermore, this work employs a fuzzy based Technique for Order Performance by Similarity to Ideal Solution approach to realize the best sampling strategy. The results have demonstrated the significance of robust optimization techniques as well as the importance of a suitable sampling strategy in coordinate metrology. This study has also established that Poisson point distribution achieved the best accuracy and the Grid point distribution had taken the least measurement time.
\end{abstract}

\section{Introduction}

Coordinate Measuring Machine (CMM) has turned up as a leading technology to accomplish inspection in manufacturing industries. This can be attributed to its high precision and accuracy. Indeed, the large-scale benefits of CMM inspection are evident only in the presence of an explicit and well-planned inspection strategy. The irregular market requirements, as well as the needs of higher productivity, have emphasized the importance of efficient and effective inspection plan in CMM [1]. The inspection planning on CMM is an arduous activity because it is made up of many functions, such as suitable probe selection, accessible part setup, the appropriate number of inspection points and their relevant distribution, optimum inspection path, and correct measurement speed $[2,3]$. Because of the inherent complexities in CMM inspection planning, its automation becomes imperative. According to Raghunandan and Venkateswara, 2008 [4], automating the steps of inspection planning not only simplifies the inspection process but also considerably minimizes the discrepancies and provides more robust and flexible plans. The entire process of an automated CMM inspection begins with the gathering of dimensions and geometrical information from the computer-aided design (CAD). This knowledge from the CAD is utilized to generate a computer-aided inspection plan (CAIP), which provides an integration link between CAD and CMM. This inspection plan is then imported into CMM software through the Dimensional Measuring Interface Standard (DMIS) for part inspection [5]. While devising the CMM inspection plan, it is crucial to consider important factors, which may influence the performance of the final part measurement. These factors may include sampling strategy, part positioning, and orientation, surface characteristics, probing system, and environmental conditions [6]. Certainly, the sampling strategy has been recognized as one of the primary aspects, which can affect the quality of measurement results $[7,8]$. The sampling strategy can be considered as an essential parameter to accurately assess the tolerance information [9]. 
The sampling strategy involves not only the selection of sample size or the number of measurement points but also their appropriate distribution on the measuring surface [10]. Even though a higher number of inspection points increase the inspection accuracy, yet they are not preferred. It is because the increased number of measurement points also inflates the inspection time and the manufacturing cost. Hence, it is necessary to perform a part inspection using the suitable sample size [11]. However, the reduction of the number of inspection points entails the proper allotment of these points. Indeed, the appropriate location of sampling points becomes crucial to obtain an accurate feature, especially when the reduced sample size is employed [12]. As reported by Barari and Mordo [13], an inadequate number of measurement points, as well as their inappropriate locations, would provide erroneous inspection results. They realized an uncertainty of $36 \%$ in the inspection results due to incorrect point distribution. The sampling strategy may also be influenced by the accuracy and surface quality of the manufactured product [14]. The manufacturing method, feature size, accuracy requirement, etc., are decisive factors in the selection of the sample size [15]. Similarly, the selection of a suitable sample size also relies upon the surface quality of the part. According to Raghunandan and Venkateswara, 2008 [4], the number of inspection points increases with an increase in surface roughness, which means the poor quality surface requires more number of measurement points as compared to good quality surfaces. Henceforth, an appropriate sampling strategy is crucial to devise a methodic CMM inspection plan and perform a valid part measurement.

The principal objective of this work focused on producing a user-friendly, manageable, and responsive sampling plan. For this purpose, different accessible sampling strategies based on existing sequences and distributions were investigated in the assessment of the flatness error. The different sample sizes, as well as diverse point distribution algorithms, were explored. The algorithms considered in this study were Hammersley, Halton-Zaremba, Poisson, Random, and Grid. The test specimens with variable surface characteristics were fabricated to determine the effect of different manufacturing signatures on the sampling strategy. Moreover, the parts with the different areas were utilized to examine the influence of part size on the sampling strategy. Finally, the Fuzzy Technique for Order Performance by Similarity to Ideal Solution (FTOPSIS) was applied to derive a robust and compliant sampling plan for the surface of the given size and quality. This problem was modeled as a multiresponse optimization problem with two responses, the accuracy and the measurement time.

\section{Literature Survey}

The sampling strategy can be defined as a plan designed to achieve appropriate sample size and precise point locations on the geometric feature being inspected using CMM [10, 16-18]. According to Li et al. [19], the exact assessment of dimension and geometrical aspects depend on numerous factors and sampling strategy is one of the most influential.
The significance of the sampling strategy was also highlighted by Ramaswami et al. [20] in their work for the optimal inspection of circularity tolerance. They determined various factors, including accessibility, convenience, simplicity, and cost, which can affect the selection of a sampling method. Certainly, an appropriate sampling method should be adopted depending on the application, especially in cases where precision and accuracy are necessary.

The implementation of a relevant sampling strategy for an enhanced CMM performance is a demanding job. In spite of the existence of abundant approaches for specifying sampling strategy, their application is constrained owing to part intricacy, the shape of the object, etc. Correspondingly, more work is needed to devise sampling strategies for the measurement of manufactured parts adequately and efficiently. According to Bosch [21], methods to achieve a suitable sampling strategy are both lacking and disorganized throughout the literature. The researchers and metrologists have been searching for and studying various methods of sampling strategy to improve the performance of CMM inspection. Indeed, the different approaches can be identified as either unguided (or blind) or adaptive sampling strategies. The two techniques differ depending on their flexibility or adjustment according to the nature of the surfaces being measured. For example, the blind sampling strategies are independent of the part shape, complexity, and surface characteristics [22]. In contrast, the adaptive (or guided) sampling strategies take into consideration the surface curvature, complexity, shape, etc., while preparing the sampling plan. The manufacturing errors resulting from the manufacturing process should also be accounted for in adaptive sampling approaches. For instance, the three-phase deviation-based sampling procedure as reported by Rossi [23] established adequate outcomes in terms of accuracy and measurement time. The holes with roundness errors induced by the boring process were analyzed in this investigation. A mathematical model was established by Yu et al. [24] to realize the suitable sampling strategy. They simulated the machining errors by superimposing the form errors on the nominal data. Subsequently, the maximum deviation between the substitute geometry and finite element model was utilized to iteratively distribute the points on the surface. A robust and flexible genetic algorithm based sampling methodology adopted by Elkott et al. [1] effectively optimized the number of sample points. This technique initially studied the surface complexity, curvature, patch size, etc., and then minimized the maximum deviation between the generated geometry and the original model. The manufacturing signatures (or the patterns) produced by the fabrication method are also useful in obtaining an appropriate sampling scheme [25]. Colosimo and Moroni [26] developed a signature model and distributed the points according to the manufacturing pattern distributed across the workpiece surface. This model recommended a larger number of points in regions with higher error as compared to the least deviated regions. Similarly, Poniatowska [27] generated a machining pattern model and determined the locations with the highest geometric deviations for the adaptive sampling plan. The most important step of this methodology was the calculation of systematic error of the complete batch and including this knowledge in producing a 
sampling plan for remaining parts in the batch. These studies have certainly displayed that adaptive sampling strategies are robust, accurate, and adequate, especially in curved and freeform surfaces. However, it should be recognized that the adaptive approaches are iterative and can become computationally complicated in case they are not implemented properly. A profound understanding of the manufacturing process, workpiece shape, and curvature are crucial in the effective implementation of the adaptive sampling scheme. There can also be a danger of collision between the measuring probe and the surface of the specimen due to the reconfiguration of the probe path at each iteration of the algorithm [28]. Moreover, most of the studies concerning the adaptive sampling strategy have depended on the simulation of pseudorandom surfaces, rather than the measurement of actual parts.

The blind sampling strategies can be defined as the methods which identify the point locations based on standard sequences, canonical distributions, user's experience or knowledge, etc. For instance, the point allocation may include strategies based on Hammersley sequence, HaltonZaremba series, Stratified based allocation, Poisson distribution, Random sampling, etc. Although they are not as effective as adaptive strategies, but they can be valuable in the inspection of prismatic and low curvature parts. It is because they are computationally less expensive and can save significant processing time. If the manufacturing process is known to fabricate components with precision, it is always convenient to implement a sampling plan based on existing distributions. However, the execution of the blind sampling strategies necessitates a proper combination of the number of points and the corresponding distribution algorithm. The workpieces with well-known manufacturing signatures can also be inspected effectively, provided appropriate sample size is combined with a suitable point allocation algorithm. Certainly, the choice for the point distribution algorithm is also critical because they are based on different assumptions and behave diversely in different applications. Therefore, prior knowledge and understanding of these algorithms are crucial to manage their performance. The correct assessment of the sample size for the corresponding allocation on the part surface has always been a mighty assignment to metrologists. Furthermore, the paucity of standard methods and systematic studies make this task more vigorous for the engineers [13]. It can be attributed to the fact that most of the work in recent times has been focusing on analyzing the different adaptive sampling strategies, irrespective of the convenience and availability of the blind sampling strategies. Nevertheless, there had been significant work occurring in this direction, which established their significance in various CMM applications. For instance, three-point distribution algorithms, including circumferential section method, generatrix method, and helix path method, were developed by Vrba et al. [29]. Likewise, Guan et al. [30] developed a spiral sampling approach for the evaluation of flatness error. The speed of the linear and the circular movement was the essential factor in spiral sampling. Similarly, Woo et al. [31] investigated three different sampling strategies based on uniform sampling, Hammersley and Halton-Zaremba sequences. They suggested that uniform sampling was not as effective as Hammersley and Halton-Zaremba due to higher sample size and error approximation. It was also mentioned that Hammersley was more accurate than the random point distribution at the given sample size. Moreover, Kim and Raman [32] introduced the concept of the priority coefficient to select the appropriate sampling strategy. They considered four sampling methods based on the Hammersley sequence, the Halton-Zaremba sequence, aligned systematic, and systematic random sampling. Each of these methods was analyzed for sample sizes $4,8,16,32$, and 64 . They concluded that systematic random sampling and the Halton-Zaremba sequence resulted in the highest accuracy of flatness evaluation. They also found aligned systematic and systematic random sampling methods with shortest inspection time as compared to Hammersley and Halton-Zaremba sequences. The spiral and Hamspi sampling methods proposed by Collins et al. [33] can be employed for the evaluation of flat and revolved surfaces. They inferred that spiral and Hamspi methods had similar point distributions as that of Hammersley sequence; however, they focused more on the origin of the workpiece. As reported by Rossi and Lanzetta [34], suitable sample size and point distribution are imperative to attain the best accuracy and shortest inspection time. It is fundamental to determine the proper sampling strategy, before form error evaluation. It is also crucial to consider the different factors, such as part size and surface characteristics, while devising the sampling strategy for the inspection process. Earlier studies have further asserted the importance of prior knowledge and information about different methods in formulating the sampling strategy.

Based on the preceding discussion, there is a need to explore the applications of different standard sequences, existing distributions, etc., in sampling strategies. It is because they are easy to use and interpret, relatively simple, highly accessible, etc. However, the performance of these available sequences or distributions needs to be enhanced using an appropriate sample size. A large number of points would unnecessarily increase the inspection time and insufficient points would degrade the measurement accuracy. It mandates the need for an optimum combination of sample size and points allocation algorithm depending on the quality and size of the surface being measured. It can be realized that a befitting consolidation of the number of points and their distribution has the competency to provide economic and accurate results. It advocates the need for an optimization approach that must consider the associated uncertainties and determine the relevant sampling plan. Henceforth, the FTOPSIS has been employed in this research to acknowledge its pertinence for acquiring a suitable sampling scheme in coordinate metrology.

\section{Sampling Methods}

In this section, different sampling methods are briefly described. The simplest methods to distribute sample points on the measuring surface are Random and Grid point distribution methods. The random point distribution 
strategy haphazardly allocates points on the inspection feature. Although it is easy to implement, most often, it is inconsistent and inadequate as it neglects many portions on the measuring surface, resulting in a cluster of points in some regions and negligible points in the other. The inconsistency in the random sampling method may have been induced due to the random point generation process. On the other hand, Grid point distribution is systematic because it distributes the sampling points on the measuring surface in a regular pattern and is one of the most accepted methods. It comprises equally spaced straightness contours in two orthogonal directions to generate a rectangular grid [35]. Grid point distribution should not be a preferred option in surfaces, which are not smooth and possess uneven or noisy texture [16]. In addition to Random and Grid point distributions, low-discrepancy sequences such as Hammersley, HaltonZaremba, and Poisson can also be utilized because they provide a good and reliable distribution of inspection points. The Hammersley method [36] is one of the most renowned sampling techniques because of its reasonably uniform point distribution. The following set of equations can be used to compute the $x$ and $y$ coordinates of the sampling points [31]. To determine the coordinates on the actual measuring surface, the $x$ and $y$ coordinates must be multiplied by the surface being inspected.

$$
\begin{aligned}
& x_{i}=\frac{i}{N}, \\
& y_{i}=\sum_{j=0}^{k-1} b_{i j} 2^{-j-1}
\end{aligned}
$$

where

$N$ represent the sample size or the total number of measurement points,

$i$ symbolizes the $i^{\text {th }}$ point and it ranges between 0 and $N-1$,

$b_{i}$ denote the binary representation of index $i$,

$b_{i j}$ represent the $j^{\text {th }}$ bit in $b_{i}$,

$j \in[0, k-1]$, and

$k$ can be defined as the total number of bits and can be computed as

$$
k=\left[\log _{2} N\right]
$$

Note that the value of $k$ should be rounded to the nearest integers greater than or equal to $\log _{2} N$. Another point distribution strategy similar to Hammersley is based on the Halton-Zaremba sequence [37]. In order to utilize HaltonZaremba for any number of sampling points, the following set of equations were implemented.

$$
\begin{aligned}
& x_{i}=\frac{i}{N}, \\
& y_{i}=\sum_{j=0}^{k-1} b_{i j}^{\prime} 2^{-j-1}
\end{aligned}
$$

$b_{i j}^{\prime}=1-b_{i j}$ if $j$ is odd and $b_{i j}^{\prime}=b_{i j}$ if $j$ is even.

Note that the value of $k$ should be rounded to the nearest integers greater than or equal to $\log _{2} N$.
These sequences can analyze the measuring surface more efficiently than the Random and Grid point distributions. They are especially useful to determine the set of points, which are more significant in the accurate evaluation of the form tolerance. The limitations of the Grid and Random sampling strategies can also be overcome by the Poisson point distribution method. It results in a more uniform distribution than the Random point distribution and is independent of any periodic behavior like Grid point distribution. It allocates the sampling points in such a way that each of the two points is at least at a specified minimum distance apart [38]. It has a peculiar property that produces a random set of points, which are rigidly arranged as well as separated by a constant minimum distance. This algorithm takes the minimum distance between points and a constant $k$ as the limit of samples to be selected, before rejection in the algorithm (typically $k=30$ ). It can be accomplished in the following three steps as described by Robert Bridson [39].

(i) Initialize an $n$-dimensional background grid and prescribe the cell size to be bounded by $\mathrm{r} / \sqrt{ } \mathrm{n}$, so that each grid cell is comprised of at most one sample point. The value of -1 indicates no sample.

(ii) Choose the initial sample, $x_{0}$, and insert it into the background grid, and initialize the active list.

(iii) If the active list is not empty, select a random index from it (say i). Generate up to $k$ points chosen uniformly from the spherical annulus between radius $r$ and $2 r$ around $x_{i}$. For each point, check if it is within distance $r$ of existing samples. If a point is located far from existing samples, select it as the next sample point and add it to the active list. If after $k$ attempts no such point is found, remove $i$ from the active list.

\section{FTOPSIS}

The implementation of a proper sampling strategy in CMM inspection is a complicated task due to the presence of numerous uncontrollable factors. These factors instill ambiguity, uncertainty, and vagueness as well as making the determination of appropriate sampling strategy a cumbersome task. Generally, the metrologists decide the sampling strategy depending on intuition or experience, which is not a convenient approach if a trade-off between inspection accuracy and measurement time is desired. Therefore, the concept of the fuzzy set theory developed by Zadeh, 1965 [40], can be combined with the Multiattribute Decision Making (MADM) method to yield accurate results and overcome any uncertainty in the system. Certainly, the effort of Bellman and Zadeh [41] can be recognized as one of the first extensions of fuzzy sets within MADM. The unification between fuzzy set theory and MADM resulted in a unique approach, now known as fuzzy MADM. This fusion is especially useful to produce decision making or optimization models that can handle incomplete and uncertain knowledge and information. In this work, the MADM method based on Fuzzy Technique for Order Performance by Similarity to Ideal Solution (FTOPSIS) has been utilized to determine 
TABLE 1: Linguistic variable and corresponding fuzzy numbers for inspection regions: (a) $90 \times 90 \mathrm{~mm}^{2}$; (b) $45 \times 45 \mathrm{~mm}^{2}$; and (c) $22.5 \times 22.5 \mathrm{~mm}^{2}$.

(a)

\begin{tabular}{lccr}
\hline Variable & Linguistic values & Range & Fuzzy numbers \\
\hline & Very Low & $\leq 0.001$ & $(0.0001,0.0003,0.0006,0.001)$ \\
CA & Low & $0.0011-0.010$ & $(0.0011,0.00407,0.00704,0.01)$ \\
& Middle & $0.011-0.025$ & $(0.011,0.0157,0.0203,0.025)$ \\
& High & $0.0251-0.05$ & $(0.0251,0.0334,0.0417,0.05)$ \\
& Very High & $>0.05$ & $(0.051,0.367,0.683,1)$ \\
Measurement time & Very Low & $\leq 1$ & $(0.1,0.3,0.7,1)$ \\
& Low & $1.1-8$ & $(1.01,3.33,5.66,8)$ \\
& Middle & $8.1-15$ & $(8.01,10.4,12.7,15)$ \\
& High & $15.1-30$ & $(15.01,20.07,25.04,30)$ \\
& Very High & $>30$ & $(30.01,36.73,43.36,50)$ \\
\hline
\end{tabular}

(b)

\begin{tabular}{|c|c|c|c|}
\hline Variable & Linguistic values & Range & Fuzzy numbers \\
\hline \multirow{5}{*}{ CA } & Very Low & $\leq 0.001$ & $(0.0001,0.0003,0.0006,0.001)$ \\
\hline & Low & $0.0011-0.01$ & $(0.0011,0.00407,0.00704,0.01)$ \\
\hline & Middle & $0.011-0.015$ & $(0.011,0.0123,0.0136,0.015)$ \\
\hline & High & $0.0151-0.0200$ & $(0.0151,0.0167,0.0184,0.02)$ \\
\hline & Very High & $>0.020$ & $(0.021,0.347,0.673,1)$ \\
\hline \multirow{5}{*}{ Measurement time } & Very Low & $\leq 1$ & $(0.1,0.3,0.7,1)$ \\
\hline & Low & $1.01-5$ & $(1.01,2.34,3.67,5)$ \\
\hline & Middle & $5.01-10$ & $(5.01,6.673,8.336,10)$ \\
\hline & High & $10.01-20$ & $(10.01,13.34,16.67,20)$ \\
\hline & Very High & $>20$ & $(20.01,30,39.99,50)$ \\
\hline
\end{tabular}

(c)

\begin{tabular}{|c|c|c|c|}
\hline Variable & Linguistic values & Range & Fuzzy numbers \\
\hline \multirow{5}{*}{$\mathrm{CA}$} & Very Low & $\leq 0.001$ & $(0.0001,0.0003,0.0006,0.001)$ \\
\hline & Low & $0.0011-0.0050$ & $(0.0011,0.0024,0.0037,0.005)$ \\
\hline & Middle & $0.0051-0.015$ & $(0.0051,0.0084,0.0117,0.015)$ \\
\hline & High & $0.0151-0.0200$ & $(0.0151,0.0167,0.0184,0.02)$ \\
\hline & Very High & $>0.020$ & $(0.021,0.347,0.673,1)$ \\
\hline \multirow{5}{*}{ Measurement time } & Very Low & $\leq 1$ & $(0.1,0.3,0.7,1)$ \\
\hline & Low & $1.01-3$ & $(1.01,1.67,2.33,3)$ \\
\hline & Middle & $3.01-8$ & $(3.01,4.673,6.336,8)$ \\
\hline & High & $8.01-15$ & $(8.01,10.34,12.67,15)$ \\
\hline & Very High & $>15$ & $(15.01,26.673,38.336,50)$ \\
\hline
\end{tabular}

TABLE 2: Relationship between CA and sample size.

\begin{tabular}{|c|c|c|c|c|c|c|}
\hline \multirow{2}{*}{ Size $\left(\mathrm{mm}^{2}\right)$} & \multirow{2}{*}{ Surface } & \multicolumn{5}{|c|}{ Percentage improvement in CA } \\
\hline & & Hammersley & Halton-Zaremba & Poisson & Random & Grid \\
\hline \multirow{3}{*}{$90 \times 90$} & 1 & 61.50 & 54.85 & 85.30 & 96.18 & 53.55 \\
\hline & 2 & 55.34 & 31.64 & 65.25 & 39.92 & 51.48 \\
\hline & 3 & 96.47 & 92.59 & 99.88 & 96.17 & 95.14 \\
\hline \multirow{3}{*}{$45 \times 45$} & 1 & 77.23 & 71.98 & 74.82 & 66.10 & 73.39 \\
\hline & 2 & 80.88 & 79.76 & 90.06 & 74.11 & 79.05 \\
\hline & 3 & 94.48 & 88.67 & 96.71 & 97.93 & 90.11 \\
\hline \multirow{3}{*}{$22.5 \times 22.5$} & 1 & 79.06 & 71.03 & 82.51 & 83.63 & 65.45 \\
\hline & 2 & 92.59 & 80.51 & 96.20 & 81.64 & 80.95 \\
\hline & 3 & 89.07 & 85.44 & 86.86 & 79.07 & 74.43 \\
\hline
\end{tabular}




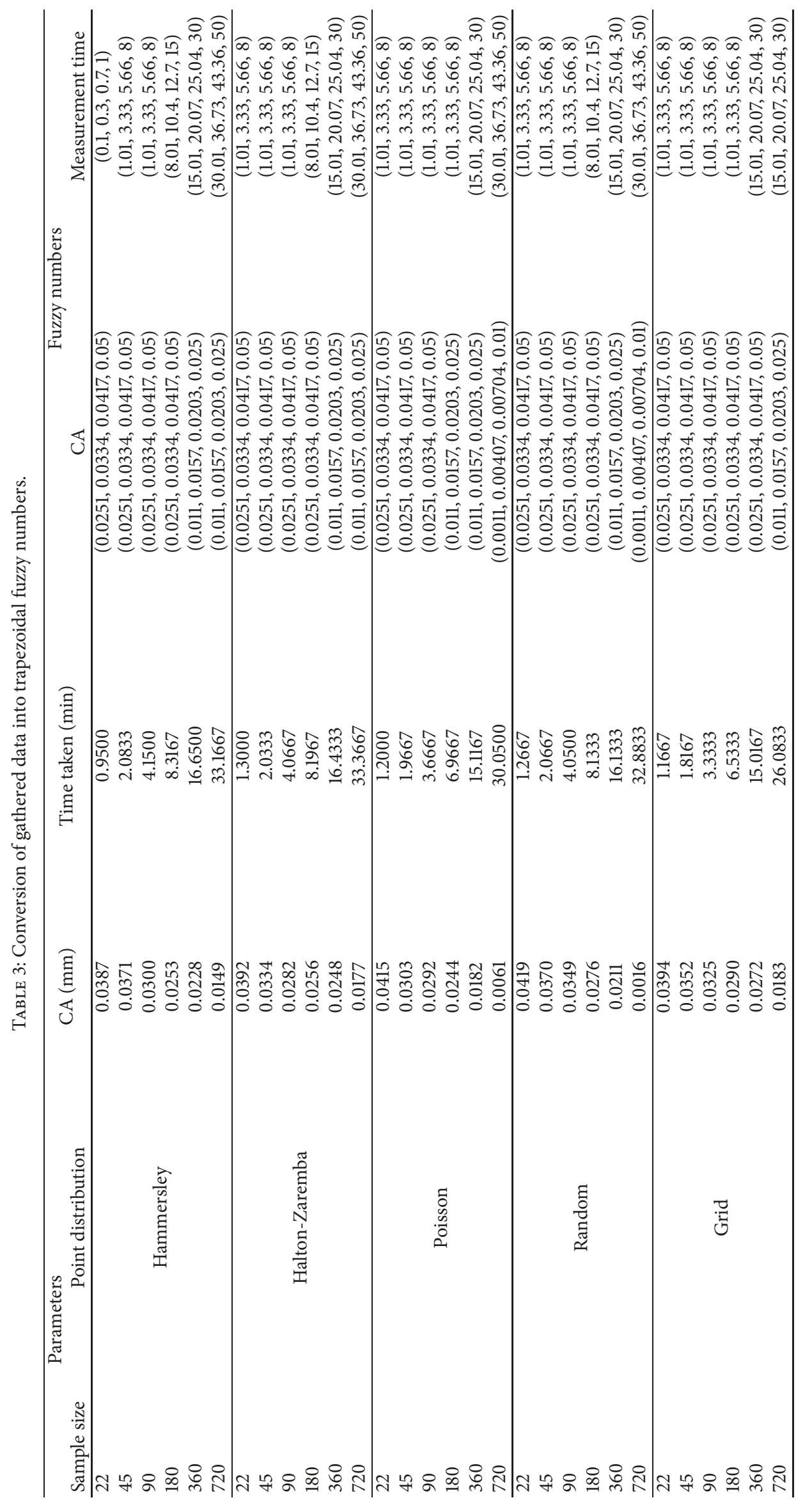


TABLE 4: Weighted normalized decision matrix.

\begin{tabular}{|c|c|c|c|c|c|c|c|}
\hline \multicolumn{8}{|c|}{ Weighted normalized fuzzy decision matrix } \\
\hline \multirow[b]{2}{*}{$\mathrm{a}_{1}$} & \multicolumn{2}{|c|}{$\mathrm{CA}$} & \multirow[b]{2}{*}{$\mathrm{d}_{1}$} & \multicolumn{4}{|c|}{ Measurement time } \\
\hline & $\mathrm{b}_{1}$ & $c_{1}$ & & $a_{2}$ & $\mathrm{~b}_{2}$ & $c_{2}$ & $\mathrm{~d}_{2}$ \\
\hline 0.011 & 0.01319 & 0.01647 & 0.02191 & 0.05 & 0.07496 & 0.15015 & 0.5 \\
\hline 0.011 & 0.01319 & 0.01647 & 0.02191 & 0.00625 & 0.00883 & 0.01502 & 0.0495 \\
\hline 0.011 & 0.01319 & 0.01647 & 0.02191 & 0.00625 & 0.00883 & 0.01502 & 0.0495 \\
\hline 0.011 & 0.01319 & 0.01647 & 0.02191 & 0.00333 & 0.00394 & 0.00481 & 0.00624 \\
\hline 0.022 & 0.02709 & 0.03503 & 0.05 & 0.00167 & 0.002 & 0.00249 & 0.00333 \\
\hline 0.022 & 0.02709 & 0.03503 & 0.05 & 0.001 & 0.00115 & 0.00136 & 0.00167 \\
\hline 0.011 & 0.01319 & 0.01647 & 0.02191 & 0.00625 & 0.00883 & 0.01502 & 0.0495 \\
\hline 0.011 & 0.01319 & 0.01647 & 0.02191 & 0.00625 & 0.00883 & 0.01502 & 0.0495 \\
\hline 0.011 & 0.01319 & 0.01647 & 0.02191 & 0.00625 & 0.00883 & 0.01502 & 0.0495 \\
\hline 0.011 & 0.01319 & 0.01647 & 0.02191 & 0.00333 & 0.00394 & 0.00481 & 0.00624 \\
\hline 0.022 & 0.02709 & 0.03503 & 0.05 & 0.00167 & 0.002 & 0.00249 & 0.00333 \\
\hline 0.022 & 0.02709 & 0.03503 & 0.05 & 0.001 & 0.00115 & 0.00136 & 0.00167 \\
\hline 0.011 & 0.01319 & 0.01647 & 0.02191 & 0.00625 & 0.00883 & 0.01502 & 0.0495 \\
\hline 0.011 & 0.01319 & 0.01647 & 0.02191 & 0.00625 & 0.00883 & 0.01502 & 0.0495 \\
\hline 0.011 & 0.01319 & 0.01647 & 0.02191 & 0.00625 & 0.00883 & 0.01502 & 0.0495 \\
\hline 0.022 & 0.02709 & 0.03503 & 0.05 & 0.00625 & 0.00883 & 0.01502 & 0.0495 \\
\hline 0.022 & 0.02709 & 0.03503 & 0.04583 & 0.00167 & 0.002 & 0.00249 & 0.00333 \\
\hline 0.055 & 0.07813 & 0.13514 & 0.5 & 0.001 & 0.00115 & 0.00136 & 0.00167 \\
\hline 0.011 & 0.01319 & 0.01647 & 0.02191 & 0.00625 & 0.00883 & 0.01502 & 0.0495 \\
\hline 0.011 & 0.01319 & 0.01647 & 0.02191 & 0.00625 & 0.00883 & 0.01502 & 0.0495 \\
\hline 0.011 & 0.01319 & 0.01647 & 0.02191 & 0.00625 & 0.00883 & 0.01502 & 0.0495 \\
\hline 0.011 & 0.01319 & 0.01647 & 0.02191 & 0.00333 & 0.00394 & 0.00481 & 0.00624 \\
\hline 0.022 & 0.02709 & 0.03503 & 0.05 & 0.00167 & 0.002 & 0.00249 & 0.00333 \\
\hline 0.055 & 0.07813 & 0.13514 & 0.5 & 0.001 & 0.00115 & 0.00136 & 0.00167 \\
\hline 0.011 & 0.01319 & 0.01647 & 0.02191 & 0.00625 & 0.00883 & 0.01502 & 0.0495 \\
\hline 0.011 & 0.01319 & 0.01647 & 0.02183 & 0.00625 & 0.00883 & 0.01502 & 0.0495 \\
\hline 0.011 & 0.01319 & 0.01647 & 0.02174 & 0.00625 & 0.00883 & 0.01502 & 0.0495 \\
\hline 0.011 & 0.01319 & 0.01647 & 0.02165 & 0.00625 & 0.00883 & 0.01502 & 0.0495 \\
\hline 0.011 & 0.01319 & 0.01647 & 0.02157 & 0.00167 & 0.002 & 0.00249 & 0.00333 \\
\hline 0.022 & 0.02709 & 0.03503 & 0.05 & 0.00167 & 0.002 & 0.00249 & 0.00333 \\
\hline
\end{tabular}

suitable and a feasible combination of a number of points and their distribution [42-44]. The Technique for Order Performance by Similarity to Ideal Solution (TOPSIS), which was introduced by Hwang and Yoon, has been the most widely employed technique among the existing MADM approaches [45]. The working of this method is based on the idea that the most appropriate alternative (or the experiment run) must have the shortest distance to the Positive Ideal Solution (PIS) and the farthermost to the Negative Ideal Solution (NIS). The PIS minimizes the negative response (or the cost attribute) and maximizes the positive response (or the benefit attribute). Similar to TOPSIS, the computation of the distance between two fuzzy numbers is one of the most important steps in FTOPSIS [46].

A simple and an effective vertex method was developed by Chen [42] to estimate the distance between two triangular (or trapezoidal) fuzzy numbers. For example, if $\tilde{x}=\left(a_{1}, b_{1}, c_{1}\right)$ and $\tilde{y}=\left(a_{2}, b_{2}, c_{2}\right)$ represent the two triangular fuzzy numbers, then the distance between these two fuzzy numbers is equal to

$$
\sqrt{\frac{1}{4}\left[\left(a_{1}-a_{2}\right)^{2}+\left(b_{1}-b_{2}\right)^{2}+\left(c_{1}-c_{2}\right)^{2}\right]}
$$

The various steps of FTOPSIS based on trapezoidal fuzzy numbers can be described as follows [47, 48].

Step 1 (representation of data using linguistic values). The implementation of a suitable sampling strategy is a complicated problem involving numerous uncertain factors and imprecise information. Therefore, the consideration of linguistic terms is quite helpful [49], especially in the problems defined in this work.

In this paper, the linguistic variables are represented using trapezoidal fuzzy numbers owing to their effectiveness, simplicity, ease of application, and general acceptance. In other words, it can be asserted that the decision matrix (or the 
TABLE 5: FPIS and FNIS.

\begin{tabular}{|c|c|}
\hline FPIS & FNIS \\
\hline $\mathrm{A}^{*}$ & $\mathrm{~A}^{-}$ \\
\hline$(0.5,0.5,0.5,0.5)$ & $(0.011,0.011,0.011,0.011)$ \\
\hline$(0.0495,0.0495,0.0495,0.0495)$ & $(0.00625,0.00625,0.00625,0.00625)$ \\
\hline$(0.0495,0.0495,0.0495,0.0495)$ & $(0.00625,0.00625,0.00625,0.00625)$ \\
\hline$(0.02191,0.02191,0.02191,0.02191)$ & $(0.0801,0.0801,0.0801,0.0801)$ \\
\hline$(0.05,0.05,0.05,0.05)$ & $(0.00167,0.00167,0.00167,0.00167)$ \\
\hline$(0.05,0.05,0.05,0.05)$ & $(0.001,0.001,0.001,0.001)$ \\
\hline$(0.0495,0.0495,0.0495,0.0495)$ & $(0.00625,0.00625,0.00625,0.00625)$ \\
\hline$(0.0495,0.0495,0.0495,0.0495)$ & $(0.00625,0.00625,0.00625,0.00625)$ \\
\hline$(0.0495,0.0495,0.0495,0.0495)$ & $(0.00625,0.00625,0.00625,0.00625)$ \\
\hline$(0.02191,0.02191,0.02191,0.02191)$ & $(0.00333,0.00333,0.00333,0.00333)$ \\
\hline$(0.05,0.05,0.05,0.05)$ & $(0.00167,0.00167,0.00167,0.00167)$ \\
\hline$(0.05,0.05,0.05,0.05)$ & $(0.001,0.001,0.001,0.001)$ \\
\hline$(0.0495,0.0495,0.0495,0.0495)$ & $(0.00625,0.00625,0.00625,0.00625)$ \\
\hline$(0.0495,0.0495,0.0495,0.0495)$ & $(0.00625,0.00625,0.00625,0.00625)$ \\
\hline$(0.0495,0.0495,0.0495,0.0495)$ & $(0.00625,0.00625,0.00625,0.00625)$ \\
\hline$(0.05,0.05,0.05,0.05)$ & $(0.00625,0.00625,0.00625,0.00625)$ \\
\hline$(0.04583,0.04583,0.04583,0.04583)$ & $(0.00167,0.00167,0.00167,0.00167)$ \\
\hline$(0.5,0.5,0.5,0.5)$ & $(0.001,0.001,0.001,0.001)$ \\
\hline$(0.0495,0.0495,0.0495,0.0495)$ & $(0.00625,0.00625,0.00625,0.00625)$ \\
\hline$(0.0495,0.0495,0.0495,0.0495)$ & $(0.00625,0.00625,0.00625,0.00625)$ \\
\hline$(0.0495,0.0495,0.0495,0.0495)$ & $(0.00625,0.00625,0.00625,0.00625)$ \\
\hline$(0.02191,0.02191,0.02191,0.02191)$ & $(0.00333,0.00333,0.00333,0.00333)$ \\
\hline$(0.05,0.05,0.05,0.05)$ & $(0.00167,0.00167,0.00167,0.00167)$ \\
\hline$(0.5,0.5,0.5,0.5)$ & $(0.001,0.001,0.001,0.001)$ \\
\hline$(0.0495,0.0495,0.0495,0.0495)$ & $(0.00625,0.00625,0.00625,0.00625)$ \\
\hline$(0.0495,0.0495,0.0495,0.0495)$ & $(0.00625,0.00625,0.00625,0.00625)$ \\
\hline$(0.0495,0.0495,0.0495,0.0495)$ & $(0.00625,0.00625,0.00625,0.00625)$ \\
\hline$(0.0495,0.0495,0.0495,0.0495)$ & $(0.00625,0.00625,0.00625,0.00625)$ \\
\hline$(0.02157,0.02157,0.02157,0.02157)$ & $(0.00167,0.00167,0.00167,0.00167)$ \\
\hline$(0.05,0.05,0.05,0.05)$ & $(0.00167,0.00167,0.00167,0.00167)$ \\
\hline
\end{tabular}

response matrix) has been described by utilizing trapezoidal fuzzy numbers. The membership function of trapezoidal fuzzy number designated by $\widetilde{T}$ and defined as $(a, b, c, d)$ can be defined as follows [50].

$$
\text { Membership function, } \mu_{\widetilde{T}}(x)= \begin{cases}0 & x \leq a \\ \frac{x-a}{b-a} & a \leq x \leq b \\ 1 & b \leq x \leq c \\ \frac{d-x}{d-c} & c \leq x \leq d \\ 0 & x \geq d\end{cases}
$$

The constants ( $a, b, c$ and $d$ ) signify the fuzziness of the assessment data [51]. It suggests that the narrower the interval $[a, d]$, the lower the fuzziness of the computing data.

Step 2 (computation of normalized fuzzy decision matrix). The normalized fuzzy decision matrix can be represented as $\widetilde{R}=\left[\widetilde{r}_{i j}\right]$. The responses (or the attributes) can be defined either as positive response (or benefit attribute) or the negative response (or cost attribute). Accordingly, the normalized fuzzy decision matrix can be obtained using the following formulation.

(i) Positive response: the higher the value of the response, the better will be the performance,

$$
\begin{aligned}
\tilde{r}_{i j}=\left\{\frac{a_{i j}}{d_{j}^{*}}, \frac{b_{i j}}{d_{j}^{*}}, \frac{c_{i j}}{d_{j}^{*}}, \frac{d_{i j}}{d_{j}^{*}}\right\}, \\
d_{j}^{*}=\max _{i}\left\{d_{i j}\right\}, j \in \text { positive response }
\end{aligned}
$$

(ii) Negative response: the lower the value of the response, the better will be the performance,

$$
\begin{aligned}
\tilde{r}_{i j}=\left\{\frac{a_{j}^{-}}{d_{i j}}, \frac{a_{j}^{-}}{c_{i j}}, \frac{a_{j}^{-}}{b_{i j}}, \frac{a_{j}^{-}}{a_{i j}}\right\}, \\
a_{j}^{-}=\min _{i}\left\{a_{i j}\right\}, j \in \text { negative response }
\end{aligned}
$$




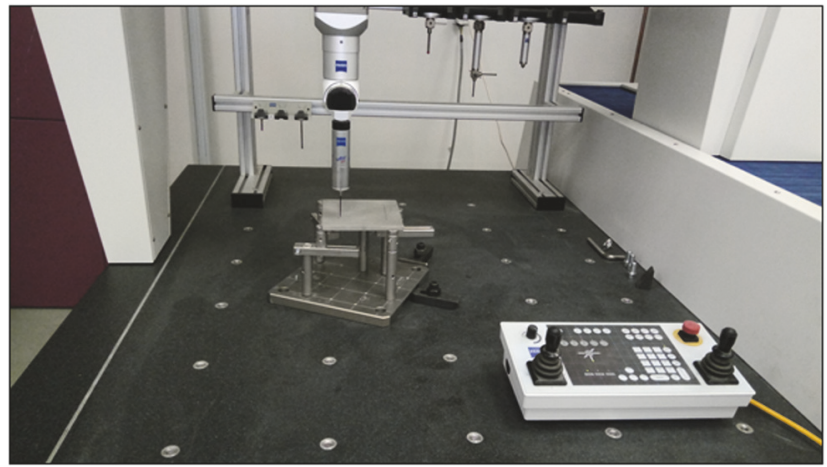

FIGURE 1: Measurement of test specimen using a CMM.

Step 3 (computation of weighted normalized decision matrix). The weighted normalized decision matrix assigns importance to different responses (or attributes) depending on the requirements. This matrix is calculated by multiplying the normalized decision matrix with the weights designated to various responses as follows.

$$
\widetilde{M}=\left(\widetilde{m}_{i j}\right), \quad \text { where } \widetilde{m}_{i j}=\widetilde{r}_{i j} * w_{j}
$$

Step 4 (computation of the fuzzy positive ideal solution (FPIS) and fuzzy negative ideal solution (FNIS)). The FPIS and FNIS are computed as follows.

$$
\begin{aligned}
& A^{*}=\left(\widetilde{m}_{1}^{*}, \widetilde{m}_{2}^{*}, m_{3}^{*}, \ldots, \widetilde{m}_{n}^{*}\right), \\
& \text { where } \widetilde{m}_{j}^{*}=\max _{i}\left\{m_{i j 4}\right\} ; \\
& A^{-}=\left(\widetilde{m}_{1}^{-}, \widetilde{m}_{2}^{-}, m_{3}^{-}, \ldots, \widetilde{m}_{n}^{-}\right), \\
& \text {where } \widetilde{m}_{j}^{-}=\min _{i}\left\{m_{i j 1}\right\} ;
\end{aligned}
$$

Step 5 (computation of the distance from each alternative to the FPIS and the FNIS).

$$
\begin{aligned}
& f_{i}^{*}=\sum_{j=1}^{n} f\left(\widetilde{m}_{i j}, \widetilde{m}_{j}^{*}\right) \\
& f_{i}^{-}=\sum_{j=1}^{n} f\left(\widetilde{m}_{i j}, \widetilde{m}_{j}^{-}\right)
\end{aligned}
$$

$f_{i}^{*}$ and $f_{i}^{-}$represent the distance from each alternative to the FPIS and the FNIS, respectively.

Step 6 (computation of the closeness coefficient $C C_{i}$ for each alternative).

$$
C C_{i}=\frac{f_{i}^{-}}{f_{i}^{*}+f_{i}^{-}}
$$

Step 7 (rank the alternatives). The alternative with highest closeness coefficient represents the best alternative.
If $\tilde{p}=\left(a_{1}, b_{1}, c_{1}, d_{1}\right)$ and $\tilde{q}=\left(a_{2}, b_{2}, c_{2}, d_{2}\right)$ represent the two trapezoidal fuzzy numbers, then

$$
\begin{aligned}
& f(\tilde{p}, \widetilde{q}) \\
& =\sqrt{\frac{1}{4}\left[\left(a_{1}-a_{2}\right)^{2}+\left(b_{1}-b_{2}\right)^{2}+\left(c_{1}-c_{2}\right)^{2}+\left(d_{1}-d_{2}\right)^{2}\right]}
\end{aligned}
$$

\section{Experimentation}

The primary goal of this work was the investigation of different sampling strategies as well as the determination of a suitable sampling plan using FTOPSIS for CMM. Five different types of point distribution algorithms, including Hammersley, Halton-Zaremba, Poisson, Random, and Grid, were employed in this study. These algorithms were chosen because of their simplicity, accessibility, convenience, and ease of application. The sample sizes preferred for the experiments were $22,45,90,180,360$, and 720, depending on the time and cost involved in the experiments. The manufacturing signatures play a significant role in the selection of a sampling strategy because they can be traced back to the manufacturing method [52]. Therefore, three different surfaces with unknown flatness and varying surface characteristics quantifying different signatures were selected as the test specimens for this experiment. For instance, the four surfaces had a surface roughness of $3.21 \mu \mathrm{m}$ (Surface 1), $4.40 \mu \mathrm{m}$ (Surface 2), and $5.80 \mu \mathrm{m}$ (Surface 3). Moreover, the three effective measurement regions were chosen to study the influence of the specimen size on flatness evaluation. The different inspection regions were identified as $90 \times 90,45 \times 45$, and $22.5 \times 22.5 \mathrm{~mm}^{2}$.

The flatness was measured using a commercial CMM (Zeiss ACCURA, 900x1200x700 $\mathrm{mm}^{3}$ ) with a tactile probe. The part setup and the CMM can be seen in Figure 1. The test specimens were mounted on the CMM machine table using a modular fixture so that the plate was stable and firm. This was done to minimize the measurement error existed during the inspection. The experiment procedure commenced with the selection of test specimens and specification of the measurement area.

Consequently, the coordinates of measurement points were generated using different algorithms in Matlab program. The coordinates were independently generated for each 

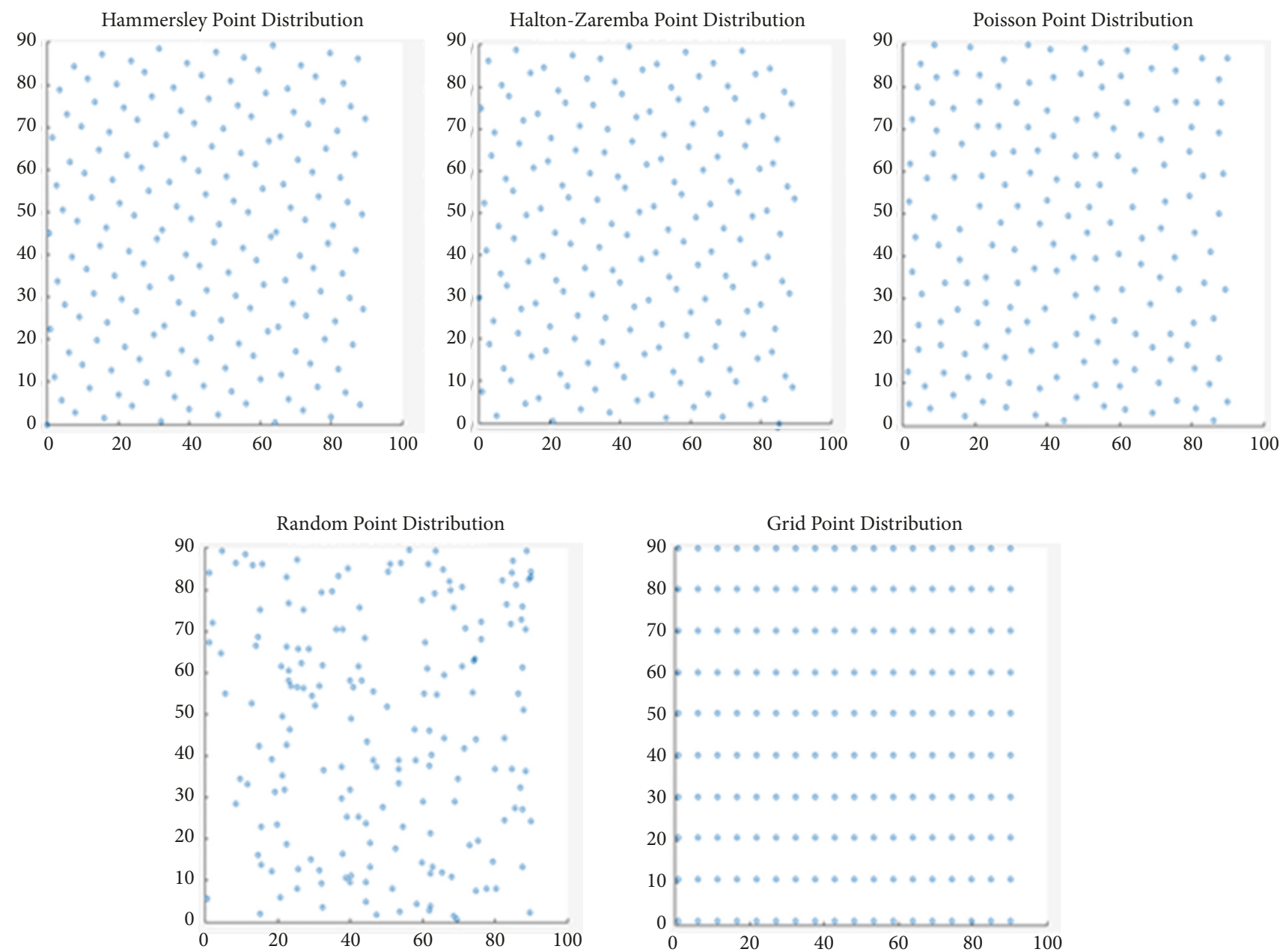

FIGURE 2: Coordinates generated using different algorithms for the measurement region of 90x90 $\mathrm{mm}^{2}$.

region, i.e., $90 \times 90,45 \times 45$, and $22.5 \times 22.5 \mathrm{~mm}^{2}$. Figure 2 shows the coordinates of inspection points for the measurement area of $90 \times 90 \mathrm{~mm}^{2}$.

The generated $(x, y)$ coordinates of the measurement point locations were provided to the CMM through an international format DMIS. The DMIS files after processing were sent to the CMM program for executing the part measurement. The flatness error was computed using the Minimum Zone form evaluation method. The procedure was repeated for all surfaces and sampling strategies. The two performance measures, i.e., closeness to actual value (CA) and the measurement time, were used to determine the competence of different point distribution algorithms. The performance measure CA was defined as the difference between the flatness error obtained using the given algorithm and the actual flatness error for the given surface. The lower value of CA represents the higher accuracy of the given algorithm. The actual value (or the true value) of the flatness error for all surfaces was calculated using a set of 45000 , 10000 , and 3000 points on sizes $90 \times 90,45 \times 45$, and $22.5 \times 22.5$ $\mathrm{mm}^{2}$ respectively. The inspection time was the time recorded for the inspection path obtained through the coordinates generated from the given sampling strategy.
Subsequently, the FTOPSIS was implemented to obtain the appropriate sampling policy. Henceforth, the different intervals (or the range) (as shown in Table 1) were defined based on the acquired data (CA and time taken) for various linguistic variables. These linguistic variables, in turn, explained the performance of the sampling strategies as well as aided in determining the most appropriate sample size and point distribution algorithm for a given surface and size.

\section{Results and Discussions}

Figures 3-5 represent the variation of CA concerning the sample size for different specimens and inspection regions. The initial observation was that of CA which depicted significant variation with the sample size and its value decreased as the number of points increased in the case of all regions and surfaces. It appears that all the algorithms except random point distribution are following a uniform pattern. There is always some probability (but not assured) to achieve the best CA with random point distribution.

It can be observed in these trends that Poisson point distribution has achieved the minimum CA. It means that 


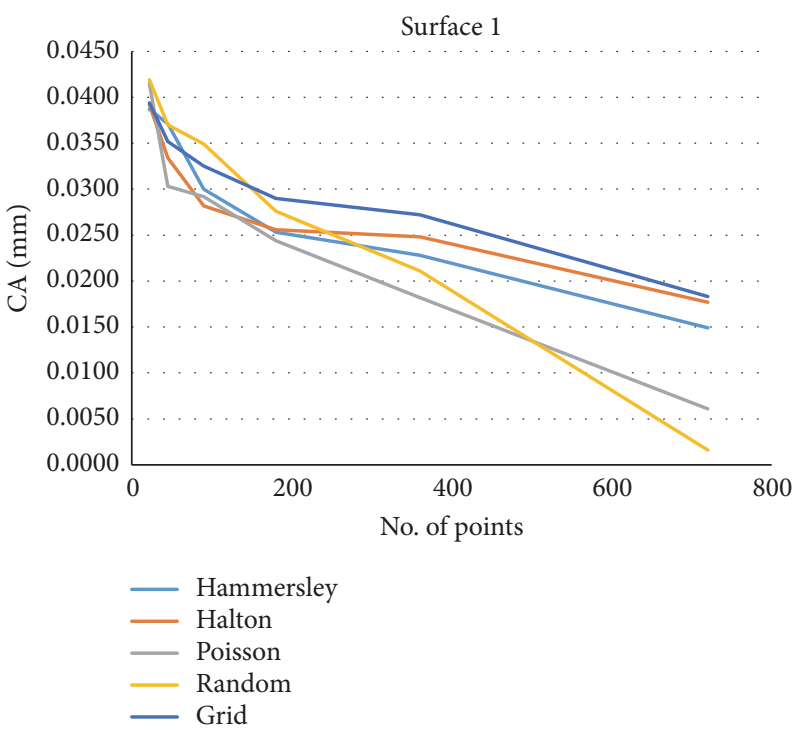

(a)

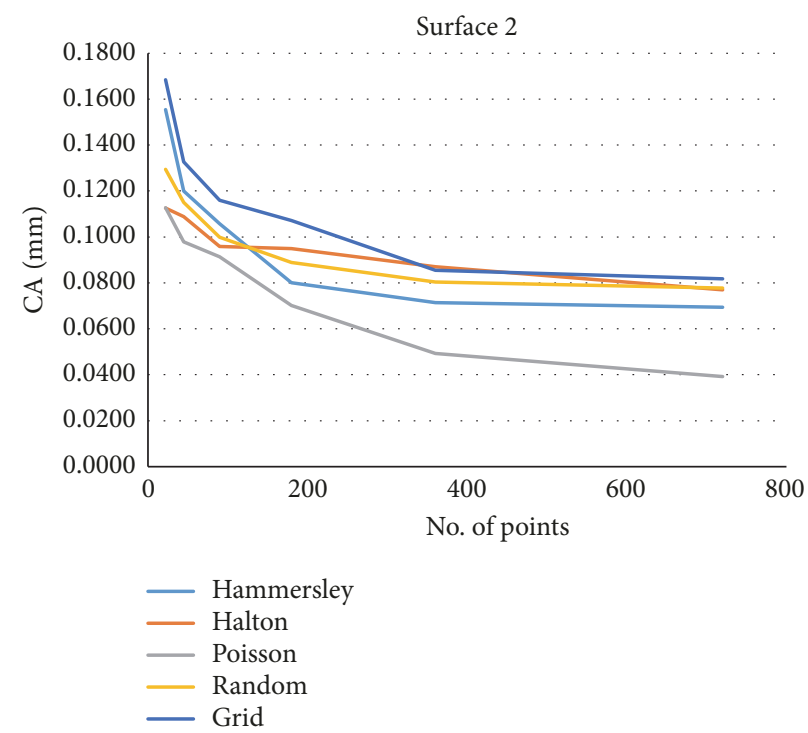

(b)

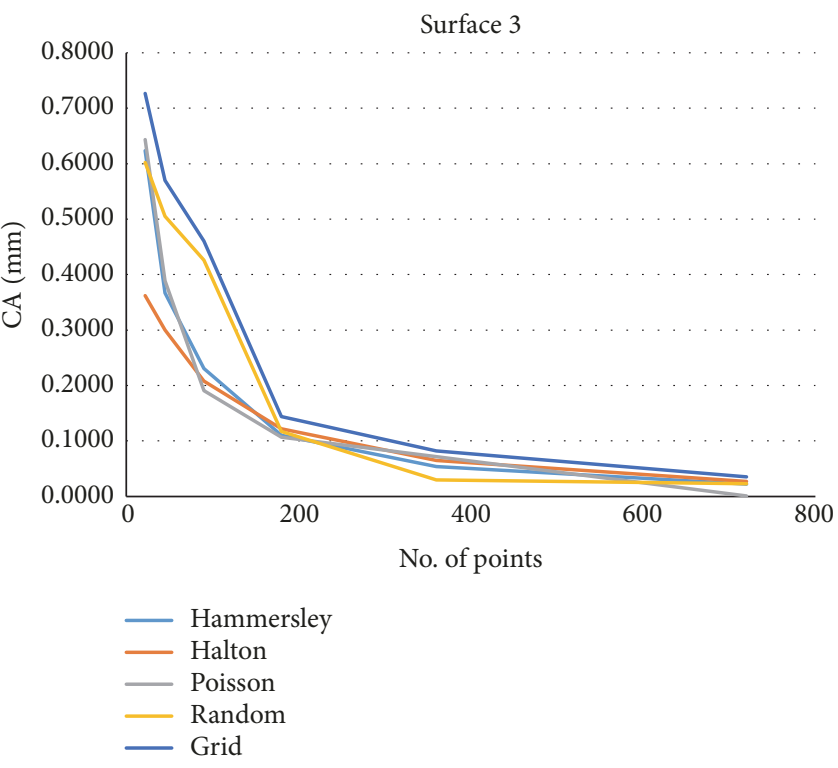

(c)

FIGURE 3: CA of algorithms for different surfaces on inspection region $90 \mathrm{x} 90 \mathrm{~mm}^{2}$.

it has achieved better accuracy as compared to other algorithms. The better performance of Poisson point distribution can be attributed to its characteristics of both randomness and systemization. It was also realized that Poisson point distribution possessed a higher CA at a lower sample size, but it decreased steeply as the number of points increased.

The Poisson point distribution algorithm was followed by the Hammersley and Halton-Zaremba point distributions in terms of CA. The CA for Hammersley algorithm improved with an increase in the sample size; however, at lower sample sizes Halton-Zaremba algorithm performed better than the Hammersley point distribution. Although the Grid point distribution has also shown significant improvement, its CA was inferior to other considered algorithms.
The subsequent step was the determination of inspection time using the coordinates obtained from different algorithms. As shown in Figure 6, the coordinates obtained from Grid point distribution provided the inspection path with the least measurement time. The Random point distribution algorithm was not consistent because in some cases, it was efficient but in others, it took a long time. The Hammersley and Halton-Zaremba algorithms resulted in the highest inspection time as compared to others. The Poisson distribution turned out to be lesser efficient as compared to the Grid point distribution.

Table 2 shows the percentage improvement in CA as the sample size was increased from 22 to 720 for different surfaces and inspection regions. It can be seen that there was 


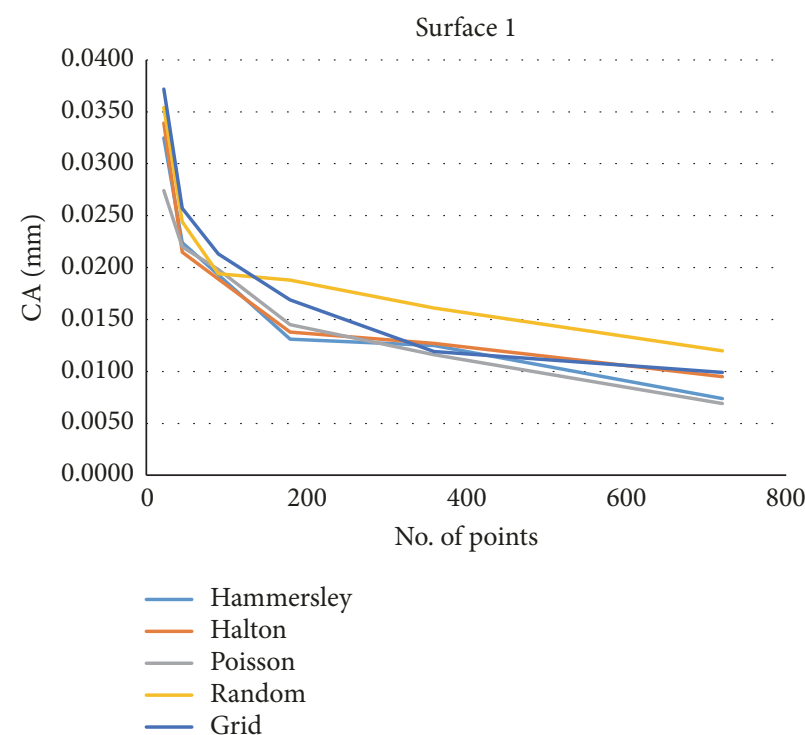

(a)

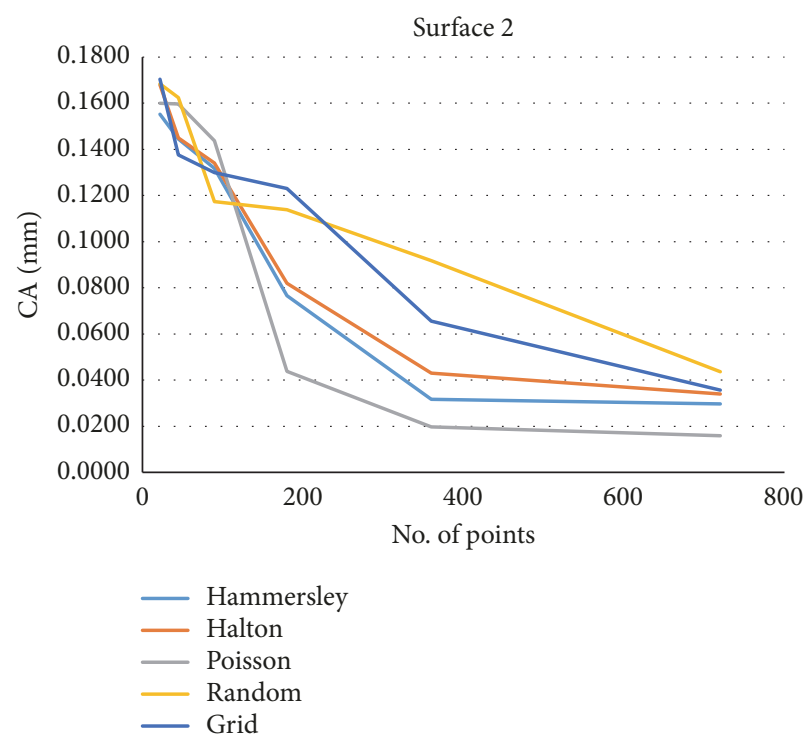

(b)

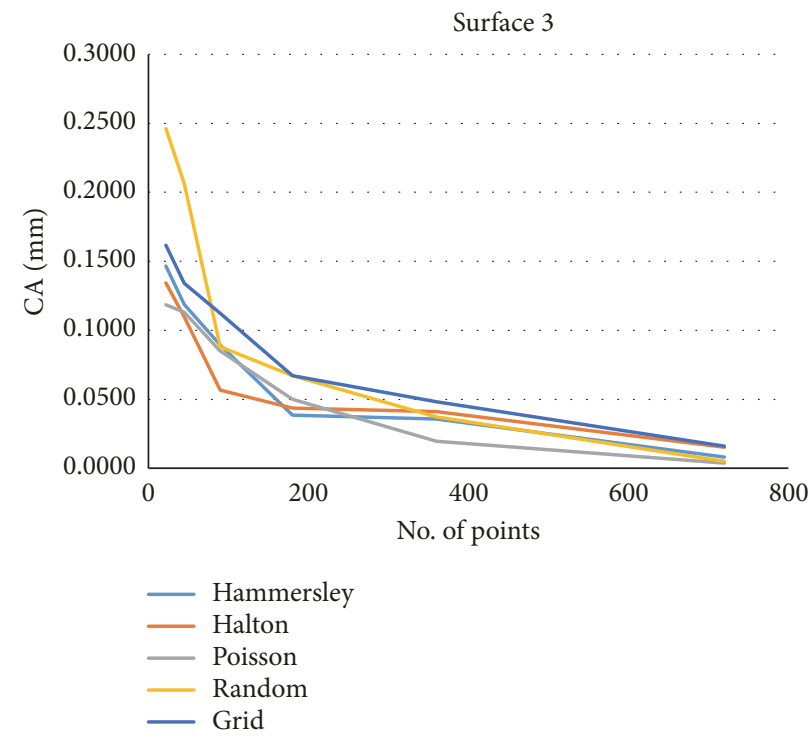

(c)

FIGURE 4: CA of algorithms for different surfaces on inspection region $45 \mathrm{x} 45 \mathrm{~mm}^{2}$.

a significant improvement in CA in all scenarios. Among all the algorithms, the Poisson point distribution had a leading performance in terms of CA. For instance, the superior performance with $65.25 \%$ improvement was observed with Poisson point distribution for Surface 2 and size 90x90 $\mathrm{mm}^{2}$. In contrast, enhancement of $55.34 \%, 31.64 \%, 39.92 \%$, and $51.48 \%$ was noticed with Hammersley, Halton-Zaremba, Random, and Grid point distributions, respectively, when the number of points increased from 22 to 720 .

The investigation also revealed a significant effect of specimen size on the flatness error. As observed in Figure 7 which represents the trend for Poisson point distribution, the CA was higher in case of large size plates for the same number of points in numerous instances. It suggests that the requirement for the number of points increases as the size of the specimen increases to achieve the same level of accuracy. A similar trend was obtained for other point distribution algorithms, where a higher sample size was needed for larger specimens. It is confirmed here that in the case of smaller inspection regions, higher accuracy can be achieved at a lesser number of points as compared to larger regions. It further indicates that the sample size can be associated with the part size to attain the desired level of measurement accuracy.

Moreover, it is shown in Figure 8 that the sample size and CA for Poisson point distribution were influenced by different surfaces (different surface characteristics). Indeed, the persistent trend was that the surface 3 possessed a higher $\mathrm{CA}$ as compared to other surfaces at a given number of inspection points. It may be attributed to its higher surface roughness $(5.80 \mu \mathrm{m})$ as compared to other surfaces. It 


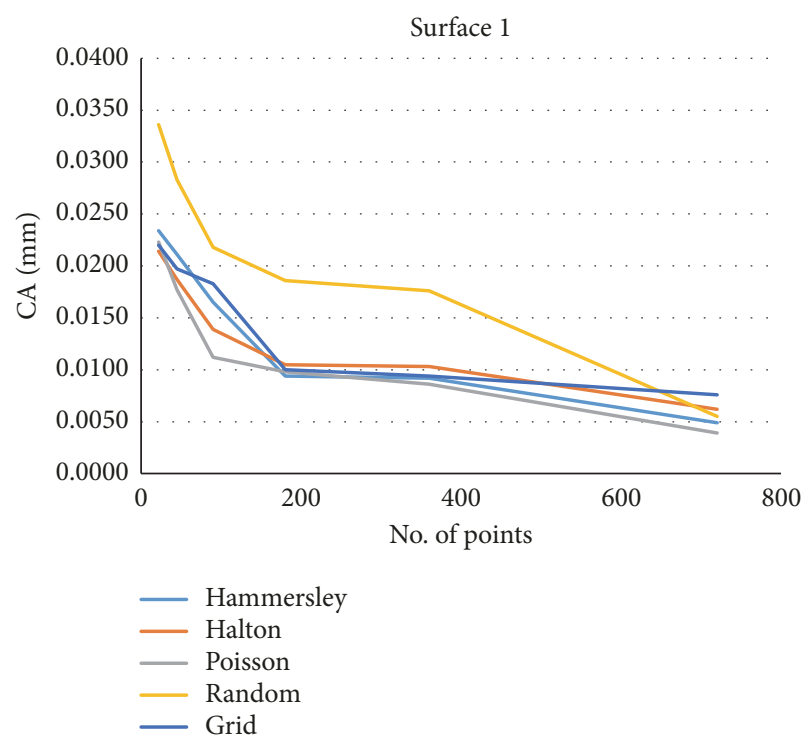

(a)

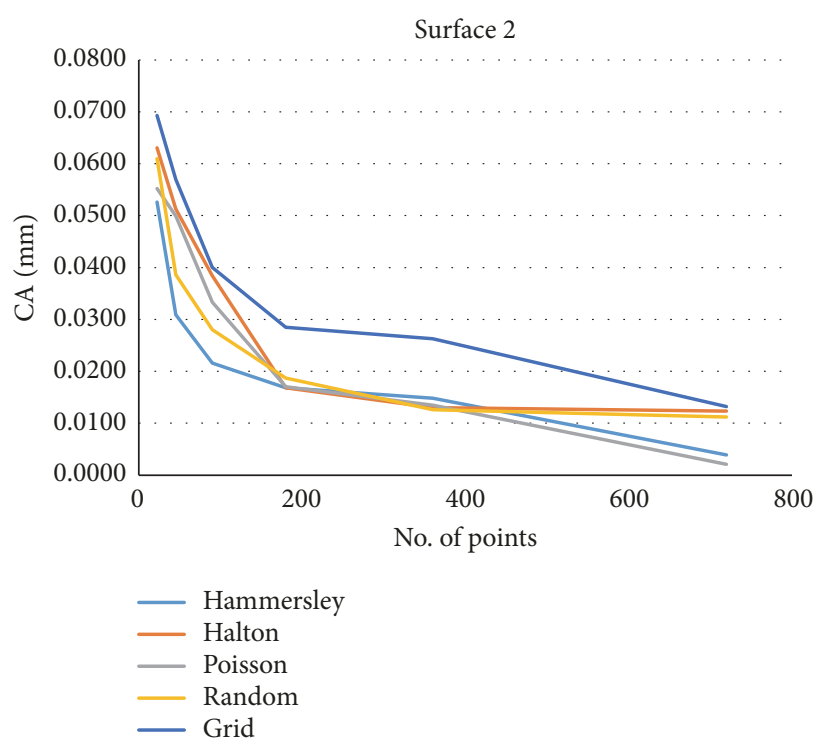

(b)

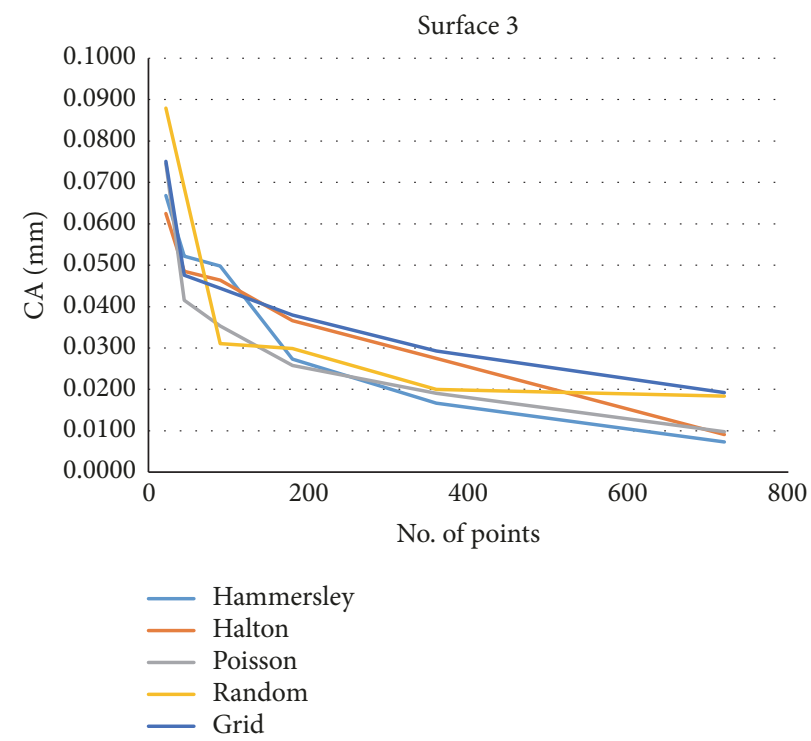

(c)

FIGURE 5: CA of algorithms for different surfaces on inspection region $22.5 \times 22.5 \mathrm{~mm}^{2}$.

suggests that the fabrication approach and the subsequently produced manufacturing signatures, as well as surface quality, play an important role in determining the sample size for the evaluation of flatness error. It was also a general trend in the case of other point distribution algorithms, where surfaces with the poor surface quality demanded a higher number of measurement points. Indeed, a smaller sample size would be sufficient to represent a good quality surface because of its lesser variations.

After analyzing the performance of different algorithms, the FTOPSIS was implemented and the following results were obtained for surface 1 with inspection region 90x90 $\mathrm{mm}^{2}$. This problem was considered as a multiresponse optimization problem with CA and measurement time as the two responses. Tables 3, 4, 5, and 6 represent the conversion of acquired data into trapezoidal fuzzy numbers, weighted normalized decision matrix, the matrix for FPIS together with FNIS, distance computations, and closeness coefficients, respectively. Note that equal importance (that is weights of 0.5 each) was assigned to the responses. The two responses were needed to be minimized (negative response); therefore, Table 1 and (6) to (14) (as discussed in Section 4) were utilized accordingly.

As shown in the above computations, the combination of sample size 180 and a Poisson point distribution algorithm provided superior results for surface 1 with a size $90 \mathrm{x}$ $90 \mathrm{~mm}^{2}$. Table 7 represents the outcomes in terms of the sampling strategy for various surfaces and respective sizes. It suggests Poisson point distribution as the most suitable algorithm owing to its tendency of both volatility and 
TABLE 6: Computation of distances and closeness coefficients.

\begin{tabular}{|c|c|c|c|}
\hline$f_{i}^{*}$ & $f_{i}^{-}$ & $f_{i}^{*}+f_{i}^{-}$ & $f_{i}^{-} /\left(f_{i}^{+}+f_{i}^{-}\right)$ \\
\hline 0.8399 & 0.2632 & 1.1030 & 0.2386 \\
\hline 0.0684 & 0.0324 & 0.1008 & 0.3210 \\
\hline 0.0684 & 0.0324 & 0.1008 & 0.3210 \\
\hline 0.0249 & 0.0146 & 0.0395 & 0.3705 \\
\hline 0.0672 & 0.0345 & 0.1017 & 0.3393 \\
\hline 0.0683 & 0.0346 & 0.1029 & 0.3363 \\
\hline 0.0684 & 0.0324 & 0.1008 & 0.3210 \\
\hline 0.0684 & 0.0324 & 0.1008 & 0.3210 \\
\hline 0.0684 & 0.0324 & 0.1008 & 0.3210 \\
\hline 0.0249 & 0.0146 & 0.0395 & 0.3705 \\
\hline 0.0672 & 0.0345 & 0.1017 & 0.3393 \\
\hline 0.0683 & 0.0346 & 0.1029 & 0.3363 \\
\hline 0.0684 & 0.0324 & 0.1008 & 0.3210 \\
\hline 0.0684 & 0.0324 & 0.1008 & 0.3210 \\
\hline 0.0684 & 0.0324 & 0.1008 & 0.3210 \\
\hline 0.0543 & 0.0514 & 0.1057 & 0.4860 \\
\hline 0.0596 & 0.0331 & 0.0926 & 0.3569 \\
\hline 0.8555 & 0.2630 & 1.1185 & 0.2351 \\
\hline 0.0684 & 0.0324 & 0.1008 & 0.3210 \\
\hline 0.0684 & 0.0324 & 0.1008 & 0.3210 \\
\hline 0.0684 & 0.0324 & 0.1008 & 0.3210 \\
\hline 0.0249 & 0.0146 & 0.0395 & 0.3705 \\
\hline 0.0672 & 0.0345 & 0.1017 & 0.3393 \\
\hline 0.8555 & 0.2630 & 1.1185 & 0.2351 \\
\hline 0.0684 & 0.0324 & 0.1008 & 0.3210 \\
\hline 0.0685 & 0.0323 & 0.1008 & 0.3207 \\
\hline 0.0685 & 0.0323 & 0.1008 & 0.3204 \\
\hline 0.0685 & 0.0323 & 0.1008 & 0.3202 \\
\hline 0.0264 & 0.0154 & 0.0418 & 0.3681 \\
\hline 0.0672 & 0.0345 & 0.1017 & 0.3393 \\
\hline
\end{tabular}

TABLE 7: Sampling schemes obtained using FTOPSIS.

\begin{tabular}{|c|c|c|c|c|c|}
\hline \multirow{2}{*}{ Surface } & \multirow{2}{*}{ Size $\left(\mathrm{mm}^{2}\right)$} & \multicolumn{2}{|c|}{ Sampling strategy } & \multirow{2}{*}{$\mathrm{CA}(\mathrm{mm})$} & \multirow{2}{*}{ Measurement time (min) } \\
\hline & & Sample size & Point distribution algorithm & & \\
\hline \multirow{4}{*}{1} & $90 \times 90$ & 180 & Poisson & 0.0244 & 6.9667 \\
\hline & \multirow{2}{*}{$45 \times 45$} & 90 & Poisson & 0.0198 & 3.6167 \\
\hline & & 90 & Random & 0.0194 & 3.4167 \\
\hline & $22.5 \times 22.5$ & 45 & Poisson & 0.0177 & 1.6500 \\
\hline \multirow{4}{*}{2} & $90 \times 90$ & 360 & Poisson & 0.0493 & 14.1667 \\
\hline & $45 \times 45$ & 360 & Poisson & 0.0198 & 14.0376 \\
\hline & \multirow{2}{*}{$22.5 \times 22.5$} & 180 & Poisson & 0.0170 & 6.6904 \\
\hline & & 180 & Random & 0.0187 & 6.9167 \\
\hline \multirow{4}{*}{3} & $90 \times 90$ & 720 & Poisson & 0.0008 & 32.1236 \\
\hline & $45 \times 45$ & 360 & Poisson & 0.0197 & 14.2667 \\
\hline & \multirow{2}{*}{$22.5 \times 22.5$} & 360 & Poisson & 0.0191 & 13.1333 \\
\hline & & 360 & Random & 0.0200 & 13.7833 \\
\hline
\end{tabular}




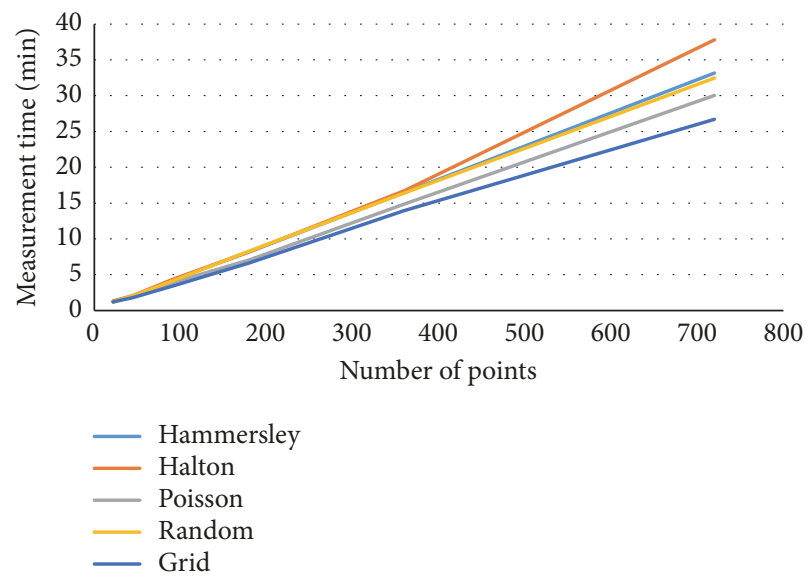

(a)

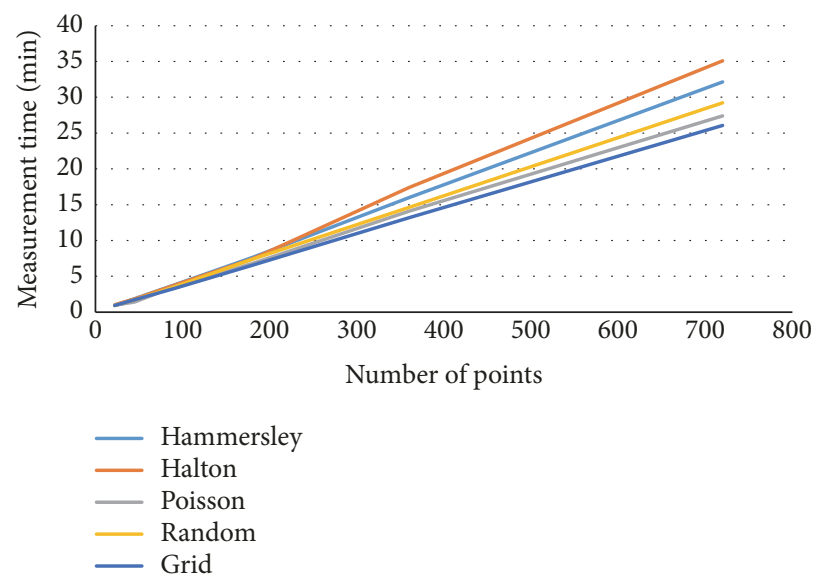

(b)

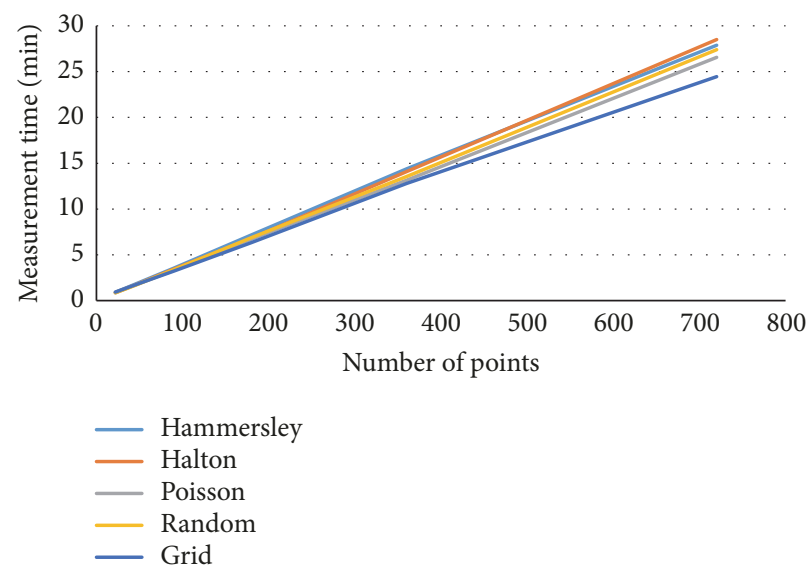

(c)

Figure 6: Measurement time for different inspection regions: (a) $90 \times 90 \mathrm{~mm}^{2}$; (b) $45 \times 45 \mathrm{~mm}^{2}$; and (c) $22.5 \times 22.5 \mathrm{~mm}^{2}$.

regularity. It can also be realized that FTOPSIS resulted in a decrease in sample size with an increase in the surface quality and a decrease in the size. At times, the Random point distribution algorithm also produced better performance, which as expected represents its arbitrary nature.

\section{Conclusion}

A quintessential sampling strategy in CMM inspection should produce meticulous measurements in lesser time. In this work, different point distribution algorithms, varying number of inspection points, and distinctive specimen sizes and surfaces have been investigated to accomplish the highest accuracy in lesser inspection time. There can be uncertainty sources which cause unreliable selection of sampling scheme. Henceforth, the appropriate selection of a sampling strategy requires a technique such as FTOPSIS which assumes fuzziness and uncertainty existed in the system. Correspondingly, this work discusses the applicability of FTOPSIS in the optimization of the sampling strategy. The FTOPSIS optimization technique certainly systemized the optimization of sampling plan with multiple responses. For instance, it has recommended a higher sample size and a Poisson point distribution algorithm (predominantly) for large and poor quality surfaces. However, the optimum results may differ in case the effects of the process generating the surface are considered. It means that the FTOPSIS will adapt the sampling plan, i.e., modify the sample size and point distribution algorithm depending on the degree of surface curvature and the condition of the surface being measured.

From the exploratory analysis, it was revealed that different algorithms have shown varying performance in terms of accuracy. The Poisson point distribution has demonstrated superior performance among the different studied algorithms. Nevertheless, the accuracy in the case of all algorithms improved with an increase in the number of inspection points. Moreover, it was noticed that the HaltonZaremba algorithm had performed better than the Hammersley point distribution at a lower number of inspection points. However, with an increase in the sample size, the performance of Hammersley was better than the Halton-Zaremba algorithm. Besides, the percentage improvement in accuracy with sample size was also explored. For example, a minimum of $65 \%$ improvement in accuracy can be expected with the Poisson point distribution algorithm, when the sample size is increased from 22 to 720 . Also, the measurement time 


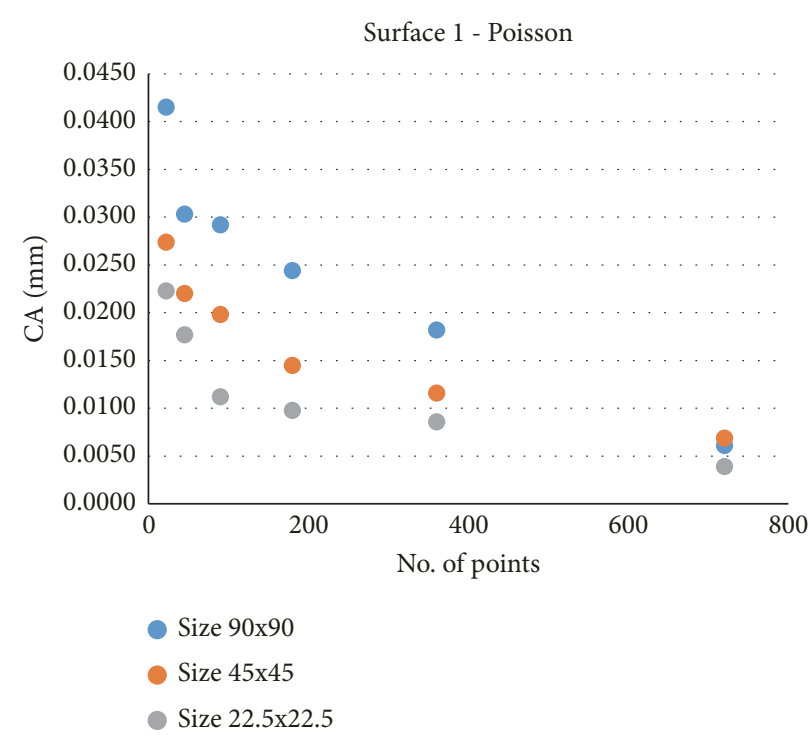

(a)

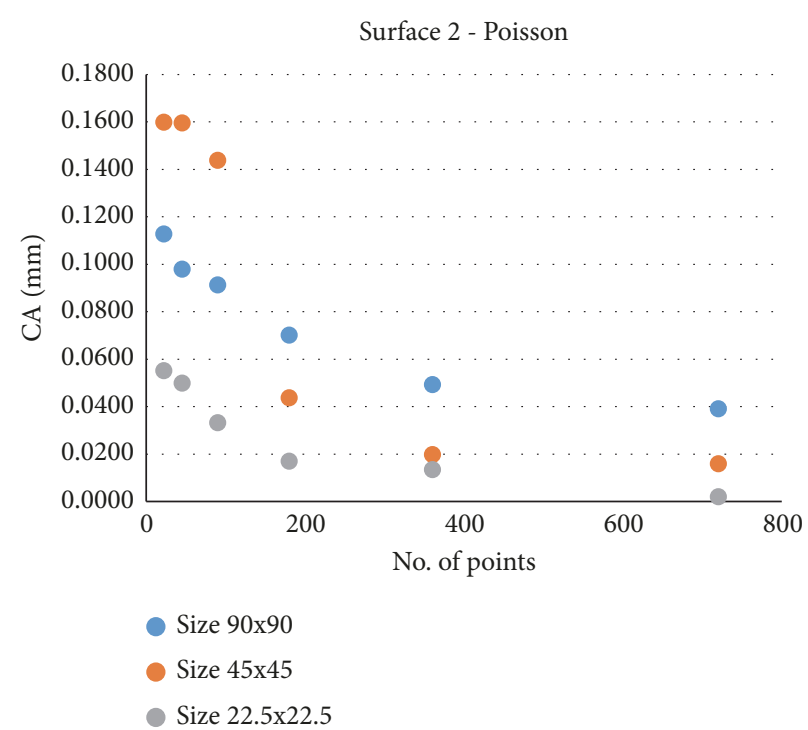

(b)

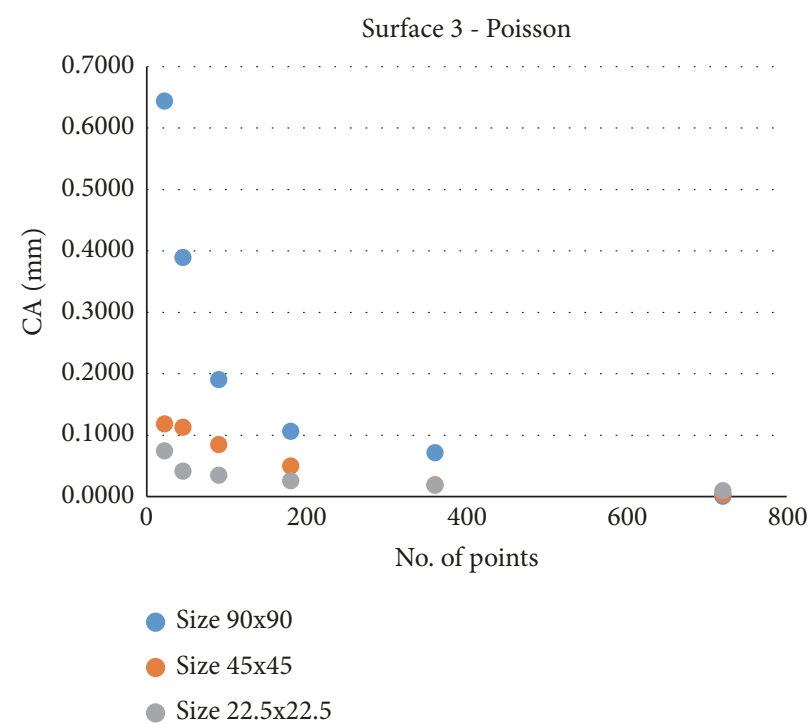

(c)

FIGURE 7: Effect of specimen size on the form error.

was also considered to establish the efficiency of different algorithms. The coordinates obtained using the Grid point distribution had taken the lowest inspection time owing to its entirely systematic pattern. The Poisson point distribution was efficient but was on the higher side as compared to the Grid point distribution because of the presence of randomness element. The Hammersley and Halton-Zaremba algorithms had taken the highest inspection time.

The best performance of Poisson point distribution in terms of both accuracy and inspection time can be credited to its properties of volatility as well as systemization. It can be established that a Poisson point distribution with appropriate sample size is generally useful in components with prismatic features and low curvatures as well as in surfaces characterized by a regular pattern of form errors. Its significance can be attributed to its many benefits, such as being easily accessible and economical both in terms of cost and time, as well as involving lesser computation. Furthermore, its performance which may deteriorate due to the influence of part manufacturing method can be sustained through various ingenious means. For example, the grid-wise Poisson point distribution can be utilized, especially in higher degree curvature surfaces or surfaces with distinct manufacturing defects or patterns. In this approach, the surface would first be divided into grids depending on the curvature profile or surface conditions and then individual grids would be filled with sample points according to Poisson point distribution. In the future, this work will be extended to utilize and adapt the Poisson point distribution for complex free form surfaces. Finally, it can be asserted that the outcomes from this analysis 


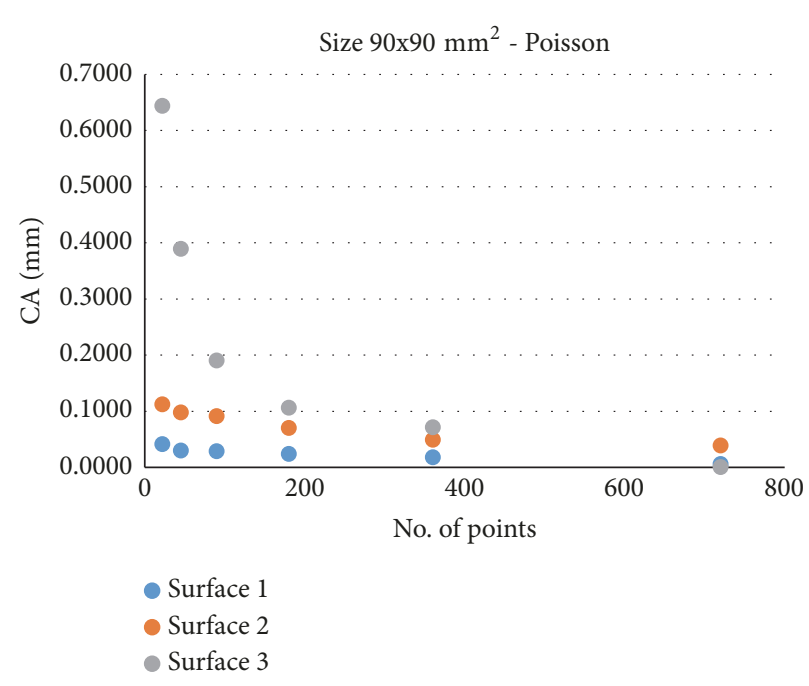

(a)

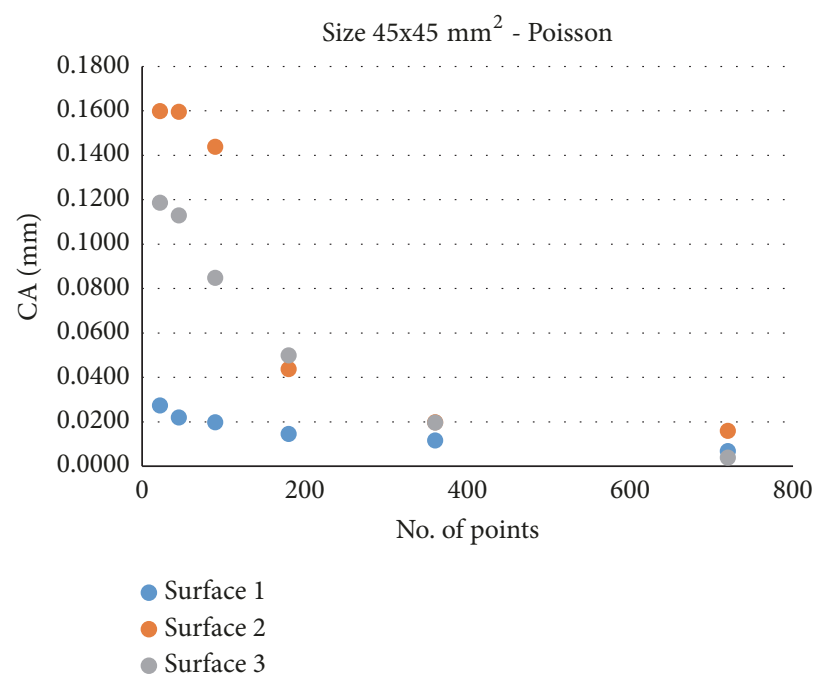

(b)

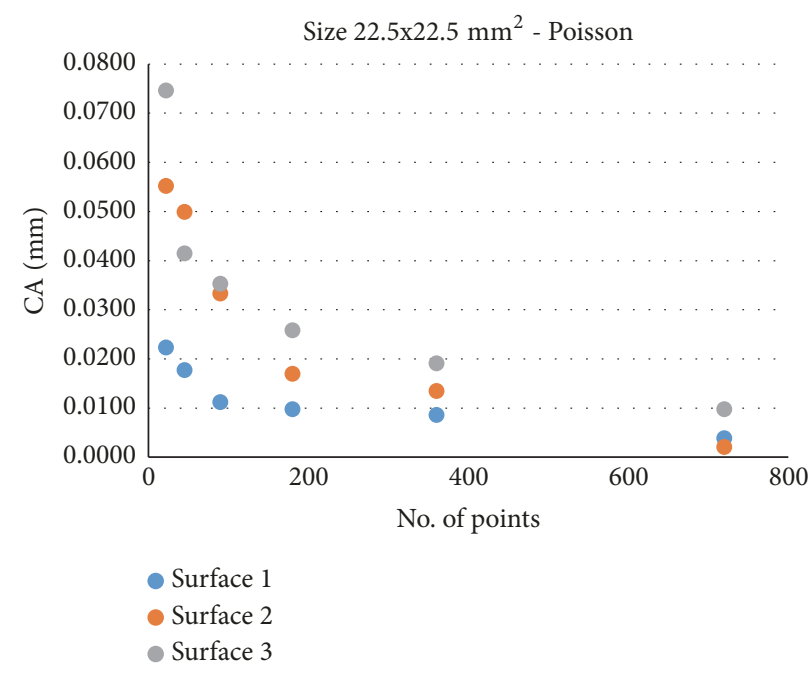

(c)

FiguRE 8: Influence of different surfaces on the form error.

can act as a guide for metrology experts or operators who require a sampling strategy for effective and efficient CMM inspection.

\section{Data Availability}

The data used to support the findings of this study are included within the article.

\section{Conflicts of Interest}

The authors declare that there are no conflicts of interest regarding the publication of this paper.

\section{Acknowledgments}

The authors are grateful to the Raytheon Chair for Systems Engineering for funding.

\section{References}

[1] D. F. Elkott, H. A. Elmaraghy, and W. H. Elmaraghy, "Automatic sampling for CMM inspection planning of free-form surfaces," International Journal of Production Research, vol. 40, no. 11, pp. 2653-2676, 2002.

[2] C. Zieman and D. Medeiros, "Automating probe selection planning for inspection on measuring machine and part setup a coordinate," International Journal of Computer Integrated Manufacturing, vol. 11, pp. 448-460, 1998.

[3] K. Sangnuan and W. S. Laosiritaworn, "Determining the optimal parameter of coordinate measuring machine with design of experiment," MATEC Web of Conferences, vol. 68, article no. 06009, p. 4, 2016.

[4] R. Raghunandan and P. Venkateswara Rao, "Selection of sampling points for accurate evaluation of flatness error using coordinate measuring machine," Journal of Materials Processing Technology, vol. 202, no. 1-3, pp. 240-245, 2008.

[5] A. Kamrani, E. A. Nasr, A. M. A. Ahmari, O. Abdulhameed, and S. H. Mian, "Feature based design approach for integrated CAD 
and computer-aided inspection planning," The International Journal of Advanced Manufacturing Technology, vol. 76, no. 9, pp. 2159-2183, 2015.

[6] A. Weckenmann, M. Knauer, and T. Killmaier, "Uncertainty of coordinate measurements on sheet-metal parts in the automotive industry," Journal of Materials Processing Technology, vol. 115, no. 1, pp. 9-13, 2001.

[7] G. Moroni and S. Petro, "Coordinate measuring machine measurement planning," in Geometric Tolerances, B. M. Colosimo and N. Senin, Eds., pp. 111-158, Springer, London, UK, 2011.

[8] Y. Z. Li, G. H. Zhang, Z. S. Liu, H. Y. Huang, S. Y. Kang, and Y. F. Peng, "Research on the measurement uncertainty of blade surface measured by coordinates measuring machines," Industrial Engineering \& Management, vol. 4, no. 4, pp. 1-5, 2015.

[9] A. B. Forbes, "Geometric tolerance assessment," NPL report DITC 210/92, National Physical Laboratory, Teddington, London, UK, October 1992.

[10] B. M. Colosimo, E. G. Moya, G. Moroni, and S. Petrò, "Statistical Sampling Strategies for Geometric Tolerance Inspection by CMM," Economic Quality Control, vol. 23, no. 1, 2008.

[11] H. Lobato, C. Ferri, J. Faraway, and N. Orchard, "Uncertainty due to experimental conditions in co-ordinate measuring machines," Proceedings of the Institution of Mechanical Engineers, Part B: Journal of Engineering Manufacture, vol. 223, no. 5, pp. 499-509, 2009.

[12] A. Jalid, S. Hariri, and N. E. Laghzale, "Influence of sample size on flatness estimation and uncertainty in three-dimensional measurement," International Journal of Metrology and Quality Engineering, vol. 6, no. 1, p. 6, 2015.

[13] A. Barari and S. Mordo, "Effect of sampling strategy on uncertainty and precision of flatness inspection studied by dynamic minimum deviation zone evaluation," International Journal of Metrology and Quality Engineering, vol. 4, no. 1, pp. 3-8, 2013.

[14] G. Lee, J. MOU, and Y. Shen, "Sampling strategy design for dimensional measurement of geometric features using coordinate measuring machine," The International Journal of Machine Tools and Manufacture, vol. 37, no. 7, pp. 917-934, 1997.

[15] Y. F. Zhang, A. Y. C. Nee, J. Y. H. Fuh, K. Neo, and H. K. Loy, "A neutral network approach to determining optimal inspection sampling size for CMM," Computer Integrated Manufacturing Systems, vol. 9, no. 3, pp. 161-169, 1996.

[16] S. Petrò, Geometric Tolerances Verification: Strategy Optimization for CMM Measurement [Ph.D. thesis], Faculty of Engineering, Department of Mechanics, Politecnico di Milano, 2008.

[17] A. Weckenmann, H. Eitzert, M. Garmer, and H. Weber, "Functionality-oriented evaluation and sampling strategy in coordinate metrology," Precision Engineering, vol. 17, no. 4, pp. 244-252, 1995.

[18] M. Ren, C. F. Cheung, L. Kong, and S. Wang, "Quantitative analysis of the measurement uncertainty in form characterization of freeform surfaces based on monte carlo simulation," in Proceedings of the CIRP, vol. 27, pp. 276-280, 2015.

[19] H. Li, X. Chen, H. Wang, Q. Yang, and Y. Chang, "Comparison of the influence of CMM measurement strategies on uncertainty evaluation," in Proceedings of SPIE: Seventh International Symposium on Precision Mechanical Measurements, L. Yu, Ed., vol. 9903, Bellingham, Wash, USA, 2016.

[20] H. Ramaswami, A. Modi, and S. Anand, "A design advisor for the optimal inspection of circularity tolerance," The International Journal of Advanced Manufacturing Technology, vol. 31, no. 9, pp. 857-870, 2007.
[21] J. A. Bosch, Coordinate Measuring Machines and Systems (Technology \& Engineering), CRC Press, Boca Raton, Fla, USA, 1995.

[22] M. Shilling, H. D. Tran, X. Huo, and H. Kim, "Determination and optimization of spatial samples for distributed measurements," in Sandia Report, Sandia National Laboratories, 2010, http://prod.sandia.gov/techlib/access-control.cgi/2010/ 107333.pdf.

[23] A. Rossi, "A form of deviation-based method for coordinate measuring machine sampling optimization in an assessment of roundness," Proceedings of the Institution of Mechanical Engineers, Part B: Journal of Engineering Manufacture, vol. 215, no. 11, pp. 1505-1518, 2006.

[24] M. Yu, Y. Zhang, Y. Li, and D. Zhang, "Adaptive sampling method for inspection planning on CMM for free-form surfaces," The International Journal of Advanced Manufacturing Technology, vol. 67, no. 9, pp. 1967-1975, 2013.

[25] J.-P. Kruth, N. V. Gestel, P. Bleys, and F. Welkenhuyzen, "Uncertainty determination for CMMs by Monte Carlo simulation integrating feature form deviations," CIRP Annals Manufacturing Technology, vol. 58, no. 1, pp. 463-466, 2009.

[26] B. M. Colosimo, G. Moroni, and S. Petrò, "A tolerance interval based criterion for optimizing discrete point sampling strategies," Precision Engineering, vol. 34, no. 4, pp. 745-754, 2010.

[27] M. Poniatowska, "Deviation model based method of planning accuracy inspection of free-form surfaces using CMMs," Measurement, vol. 45, no. 5, pp. 927-937, 2012.

[28] G. Moroni and S. Petrò, "Geometric Inspection Planning as a Key Element in Industry 4.0," in Proceedings of the $3 \mathrm{rd}$ International Conference on the Industry 4.0 Model for Advanced Manufacturing (AMP 2018), J. Ni, V. Majstorovic, and D. Djurdjanovic, Eds., Lecture Notes in Mechanical Engineering, Springer, Cham, Switzerland, 2018.

[29] I. Vrba, R. Palencar, M. Hadzistevic, B. Strbac, and J. Hodolic, "The influence of the sampling strategy and the evaluation method on the cylindricity error on a coordinate measurement machine," Journal of Production Engineering, vol. 16, no. 2, pp. 53-56, 2013.

[30] P. Guan, T. B. Yu, and W. S. Wang, "Research on Flatness Error Measurement of Revolving Body End-Face," Applied Mechanics and Materials, vol. 44-47, pp. 4002-4006, 2010.

[31] T. Woo, R. Liang, C. Hsieh, and N. Lee, "Efficient sampling for surface measurements," Journal of Manufacturing Systems, vol. 14, no. 5, pp. 345-354, 1995.

[32] W. Kim and S. Raman, "On the selection of flatness measurement points in coordinate measuring machine inspection," The International Journal of Machine Tools and Manufacture, vol. 40, no. 3, pp. 427-443, 2000.

[33] C. E. Collins, E. B. Fay, J. A. Aguirre-Cruz, and S. Raman, "Alternate methods for sampling in coordinate metrology," Proceedings of the Institution of Mechanical Engineers, Part B: Journal of Engineering Manufacture, vol. 221, no. 6, pp. 10411052, 2016.

[34] A. Rossi and M. Lanzetta, "Optimal blind sampling strategy for minimum zone roundness evaluation by metaheuristics," Precision Engineering, vol. 37, no. 2, pp. 241-247, 2013.

[35] S. Lakota and A. Görög, "Flatness measurement by multipoint methods and by scanning methods," Ad Alta: Journal of Interdisciplinary Research, vol. 1, no. 1, pp. 124-127, 2011.

[36] J. M. Hammersley and D. Handscomb, Monte Carlo Methods, John Wiley and Sons, New York, NY, USA, 1964. 
[37] J. H. Halton and S. K. Zaremba, "The extreme and L2 discrepancies of some plane sets," Monatshefte for mathematik, vol. 73, no. 4, pp. 316-328, 1969.

[38] T. R. Jones, "Efficient generation of Poisson-disk sampling patterns," Journal of Graphics Tools, vol. 11, no. 2, pp. 27-36, 2006.

[39] R. Bridson, "Fast Poisson disk sampling in arbitrary dimensions," in Proceedings of the ACM SIGGRAPH Sketches (SIGGRAPH '07), San Diego, Calif, USA, August 2007.

[40] L. A. Zadeh, "The concept of a linguistic variable and its application to approximate reasoning-I," Information Sciences, vol. 8, pp. 199-249, 1975.

[41] R. E. Bellman and L. A. Zadeh, "Decision-making in a fuzzy environment," Management Science, vol. 17, no. 4, pp. B141-B164, 1970.

[42] C. T. Chen, "Extensions of the TOPSIS for group decisionmaking under fuzzy environment," Fuzzy Sets and Systems, vol. 114, no. 1, pp. 1-9, 2000.

[43] L. Shen, L. Olfat, K. Govindan, R. Khodaverdi, and A. Diabat, "A fuzzy multi criteria approach for evaluating green supplier's performance in green supply chain with linguistic preferences," Resources, Conservation \& Recycling, vol. 74, pp. 170-179, 2013.

[44] A. Awasthi, S. S. Chauhan, and S. K. Goyal, "A multi-criteria decision making approach for location planning for urban distribution centers under uncertainty," Mathematical and Computer Modelling, vol. 53, no. 1-2, pp. 98-109, 2011.

[45] C. L. Hwang and K. P. Yoon, "Multiple attribute decision making: methods and applications," in A State-of-the-Art Survey, vol. 186, Springer, Berlin, Germany, 1981.

[46] S. Pattnaik, M. Priyadarshini, K. Mahapatra, D. Mishra, and S. Panda, "Multi objective optimization of EDM process parameters using fuzzy TOPSIS method," in Proceedings of the 2015 International Conference on Innovations in Information,Embedded and Communication Systems (ICIIECS), pp. 1-5, Coimbatore, India, March 2015.

[47] C. T. Chen, C. T. Lin, and S. F. Huang, "A fuzzy approach for supplier evaluation and selection in supply chain management," International Journal of Production Economics, vol. 102, no. 2, pp. 289-301, 2006.

[48] S. Nadaban, S. Dzitac, and I. Dzitac, "Fuzzy topsis: a general view," Procedia Computer Science, vol. 91, pp. 823-831, 2016.

[49] D. Yong, "Plant location selection based on fuzzy TOPSIS," The International Journal of Advanced Manufacturing Technology, vol. 28, pp. 839-884, 2006.

[50] M. Savitha and G. Mary, "New methods for ranking of trapezoidal fuzzy numbers," Advances in Fuzzy Mathematics, vol. 12, pp. 1159-1170, 2017.

[51] G. S. Liang and M. J. J. Wang, "Fuzzy multi-criteria decisionmaking method for facility site selection," International Journal of Production Research, vol. 29, no. 11, pp. 2313-2330, 1991.

[52] G. Moroni, W. Polini, and M. Rasella, "Manufacturing signatures and CMM sampling strategies," in Proceedings of the ASPE Summer Topical Meeting on Coordinate Measuring Machines, pp. 57-62, Charlotte, North Carolina, USA, 2003. 


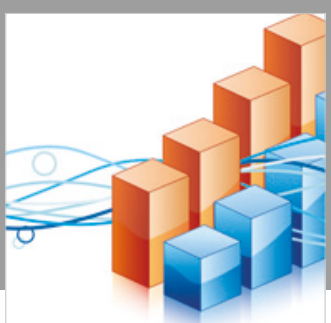

Advances in

Operations Research

\section{-n-m}
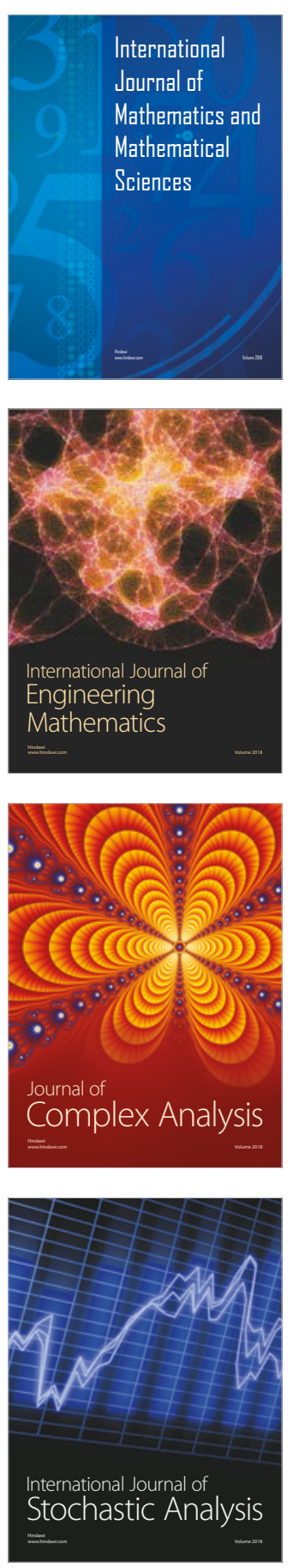
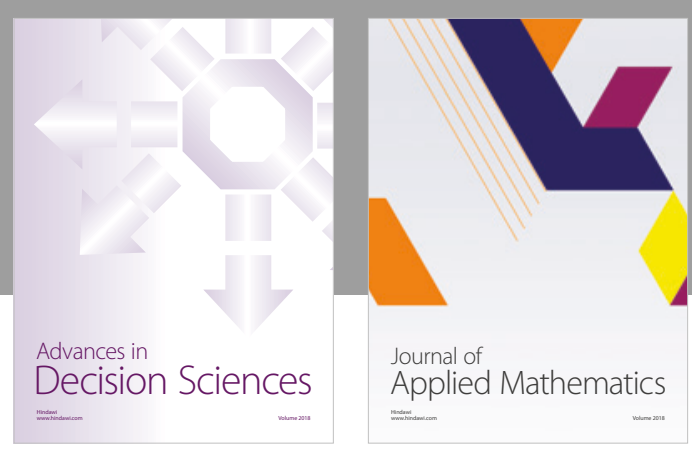

Journal of

Applied Mathematics
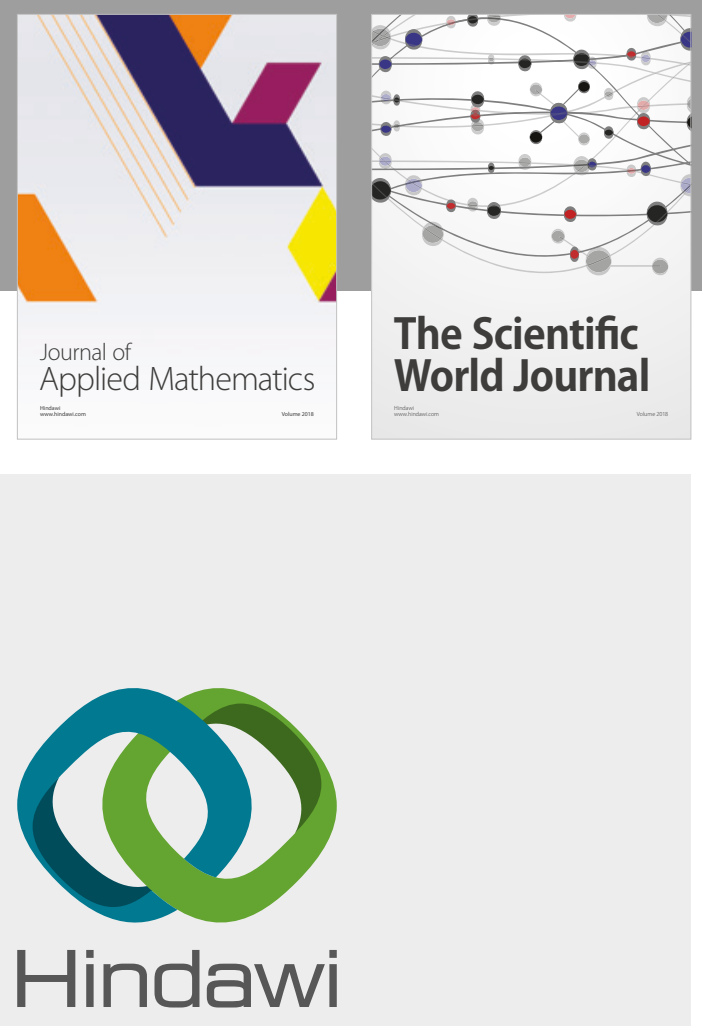

Submit your manuscripts at

www.hindawi.com

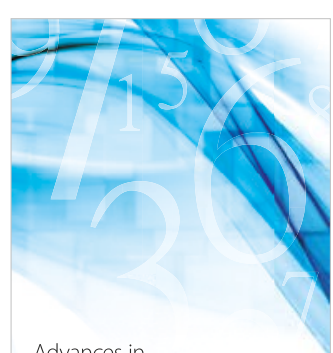

Advances in
Numerical Analysis
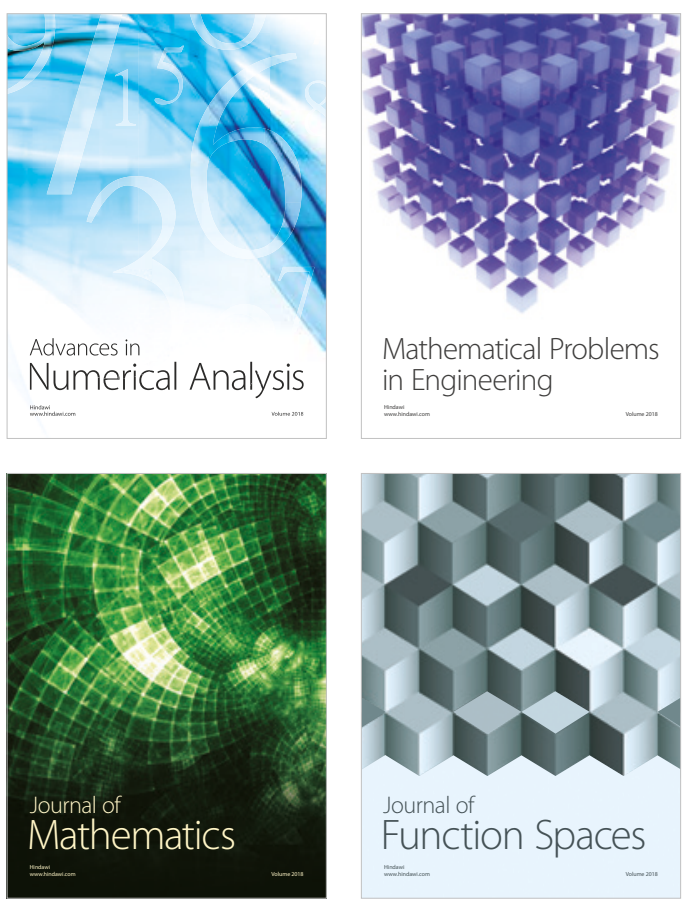

Mathematical Problems in Engineering

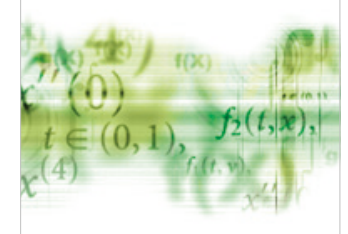

International Journal of

Differential Equations

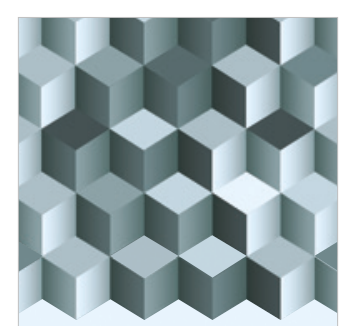

Journal of

Function Spaces

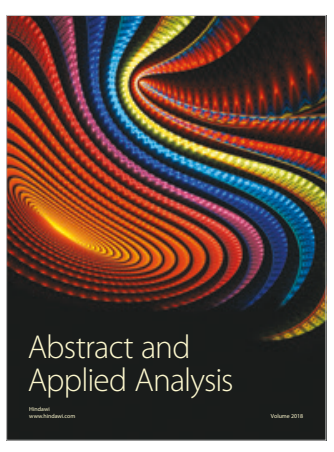

The Scientific

World Journal

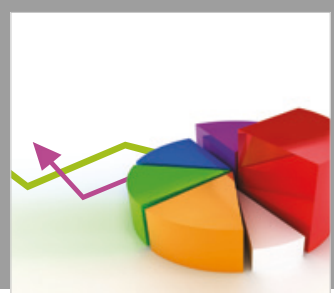

Journal of

Probability and Statistics
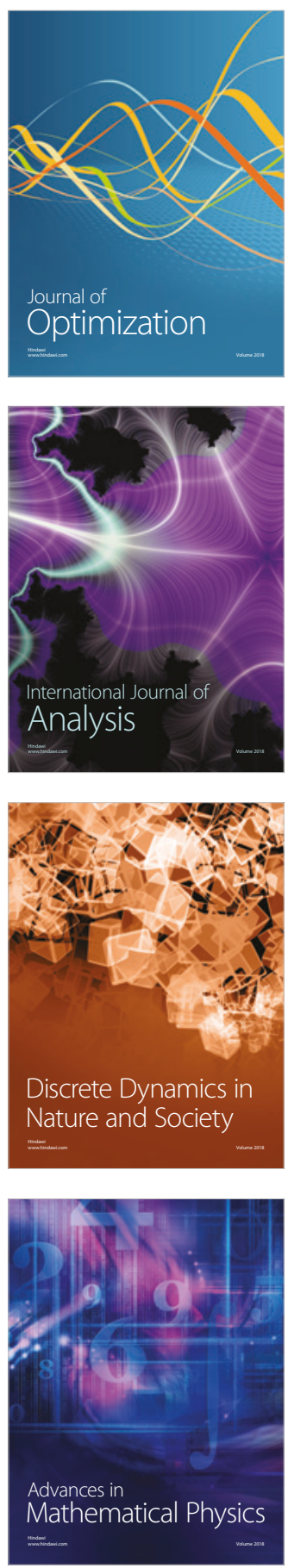